\title{
Integrating Proteomes for Lung Tissues and Lavage Reveals Pathways That Link Responses in Allergen-Challenged Mice
}

Thomas H. Mahood, ${ }^{\bigcirc}$ Christopher D. Pascoe, ${ }^{\bigcirc}$ Tobias K. Karakach, Aruni Jha, Sujata Basu, Peyman Ezzati, Victor Spicer, Neeloffer Mookherjee, and Andrew J. Halayko*

Cite This: ACS Omega 2021, 6, 1171-1189

Read Online

ABSTRACT: To capture interplay between biological pathways, we analyzed the proteome from matched lung tissues and bronchoalveolar lavage fluid (BALF) of individual allergen-naive and house dust mite (HDM)-challenged BALB/c mice, a model of allergic asthma. Unbiased label-free liquid chromatography with tandem mass spectrometry (LC-MS/MS) analysis quantified 2675 proteins from tissues and BALF of allergen-naive and HDM-

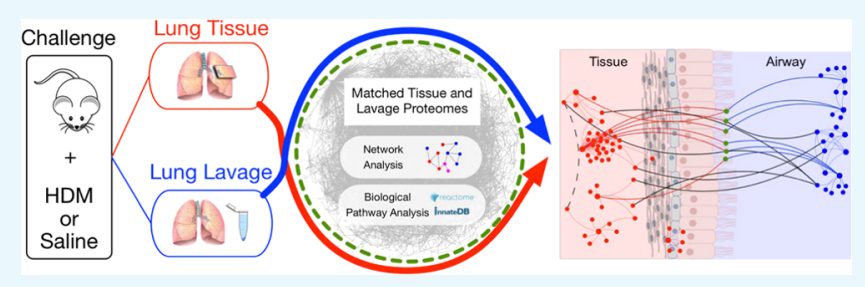
exposed mice. In comparing the four datasets, we found significantly greater diversity in proteins between lung tissues and BALF than in the changes induced by HDM challenge. The biological pathways enriched after allergen exposure were compartment-dependent. Lung tissues featured innate immune responses and oxidative stress, while BALF most strongly revealed changes in metabolism. We combined lung tissues and BALF proteomes, which principally highlighted oxidation reduction (redox) pathways, a finding influenced chiefly by the lung tissue dataset. Integrating lung and BALF proteomes also uncovered new proteins and biological pathways that may mediate lung tissue and BALF interactions after allergen challenge, for example, B-cell receptor signaling. We demonstrate that enhanced insight is fostered when different biological compartments from the lung are investigated in parallel. Integration of proteomes from lung tissues and BALF compartments reveals new information about protein networks in response to environmental challenge and interaction between intracellular and extracellular processes.

\section{INTRODUCTION}

The biological underpinnings of chronic inflammation in asthma involve a complex molecular interplay between structural cells, recruited inflammatory cells, and the extracellular mediators that they release. Understanding this interplay is important to understand pathobiology and support preclinical research to develop new therapies. Mice exposed to an allergen are a mainstay for asthma research, and a common approach is to challenge animals with inhaled house dust mite (HDM). HDM is an aeroallergen that is clinically relevant and is a complex stimulus with multiple immunogens and stressors, including fungal spores, bacterial endotoxins, lipid-binding proteins, and proteases. ${ }^{1,2}$ Repeated HDM challenge induces pathophysiological symptoms that are hallmarks of human asthma. ${ }^{3}$ However, the scope and complexity of the interplay between lung cells, autocrine and paracrine pathways, and the integrated signaling networks associated with the pathobiology that determines the efficacy of preclinical therapeutics has not been refined.

To understand complex disease mechanisms, a number of omics technologies have been employed, including proteomics. To the best of our knowledge, most studies examine the proteome of individual biological compartments, including airway spaces, collected as bronchoalveolar lavage fluid (BALF) or sputum, and lung tissues from asthmatic patients and murine models of the disease. ${ }^{4-6}$ These studies have been important for endotyping patients and animal models, identifying biomarkers of disease, and providing direction for new therapeutic strategies. However, analysis of these biological compartments in isolation can only partially identify the molecular networks and biomarkers that are critical for disease expression in a complex system like the lung. Nonetheless, understanding the molecular systems that determine the interaction between secreted proteins in the lung and the pathways that are regulated in the resident cells of the lung has not been fully established in conventional proteomics studies.

To address this knowledge gap, we used unbiased proteomics and unsupervised exploratory data analysis to establish a molecular signature in matched lung tissues and BALF from individual mice. We then combined these to create integrated proteomes that allow unique signals from each sample type to be identified and the nature of the interactions between biological compartments to be deciphered in

Received: September 1, 2020

Accepted: November 4, 2020

Published: January 5, 2021 


\section{A}

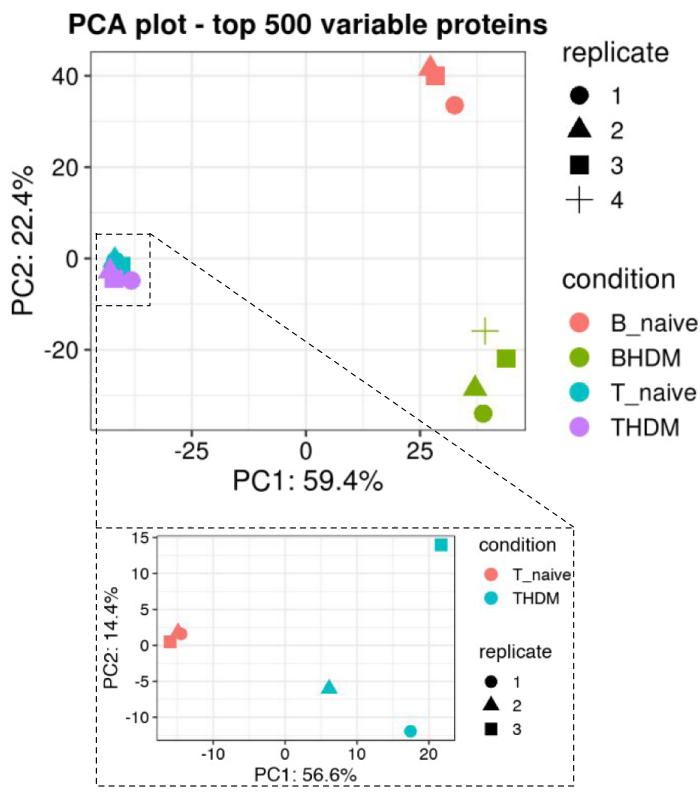

B

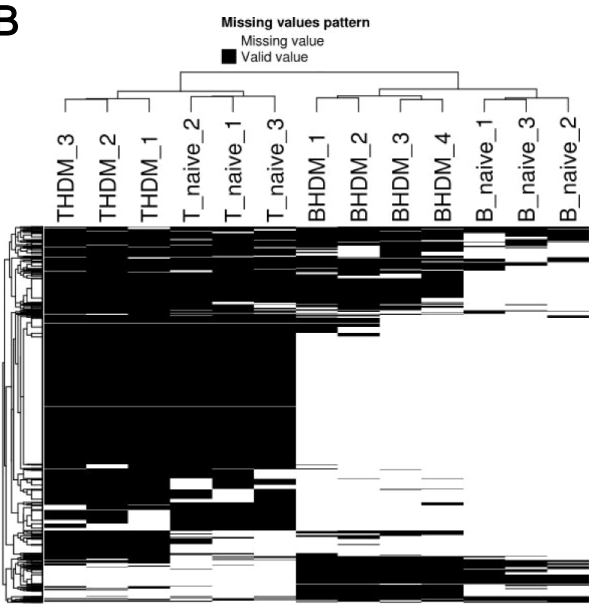

C
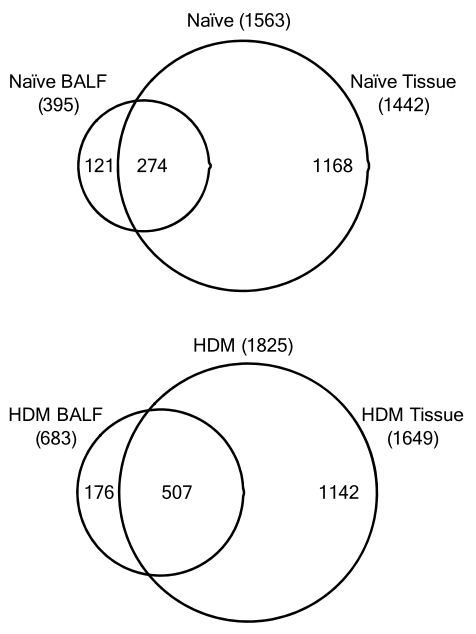

D

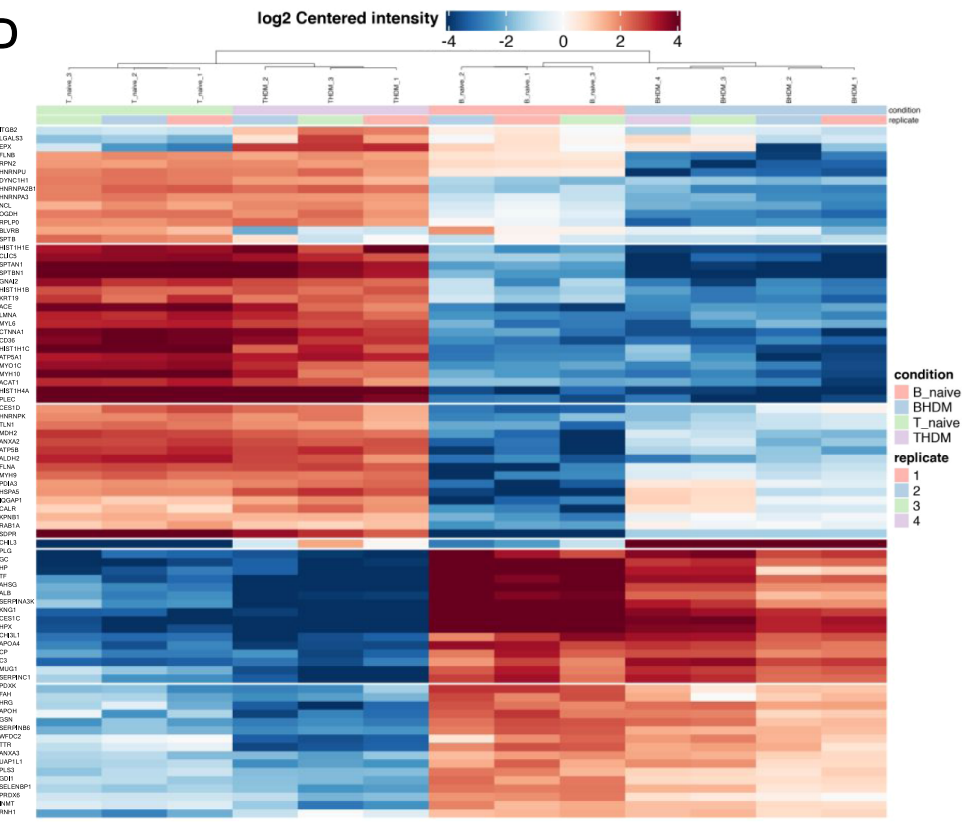

Figure 1. Characterization of the distinct proteomic signatures of the lung tissue and BALF. (A) PCA of the top 500 most variable proteins reveals that BALF and tissue datasets have greater variation (59.4\%) than the datasets comparing allergen-naive and HDM-challenged samples (22.4\%). Note that variability between the highly divergent tissue and BALF datasets masks the differences between allergen-naive and HDM-exposed lung tissue, as shown in the figure inset (PCA of the top 500 most variable proteins). (B) Differences between BALF and tissue samples are driven by missing proteins in the BALF compartment, as shown by $k$-means clustering heatmap of detectable proteins. (C) Distribution of Uniprot ID's across lung tissues and BALF in both naïe and HDM-exposed mice. (D) $k$-Means clustering heatmap showing protein abundance differences between lung tissue and BALF from allergen naive and HDM mice. Red: high abundance, blue: low abundance. Abbreviations used: house dust mite (HDM), bronchial alveolar lavage fluid (BALF), and principal component analysis (PCA).

describing the response to HDM challenge. We found that the proteomes of lung tissues and of BALF are distinct and that inhaled HDM challenge induces unique alterations in each compartment. Moreover, by integrating proteomes we uncovered novel signaling networks that were not evident from individual proteome datasets of lung tissues or BALF.

\section{RESULTS}

Lung Function and Differential Cell Count Analysis. HDM challenge of adult female BALB/c mice (2 weeks, 5 times weekly) resulted in pathophysiological features that are consistent with human asthma. This included significant increases in airway resistance, tissue elastance, and tissue resistance in response to methacholine challenge (Supporting 
A

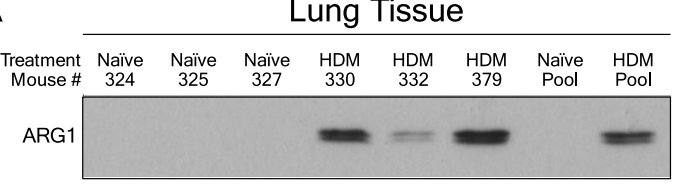

CLCA1

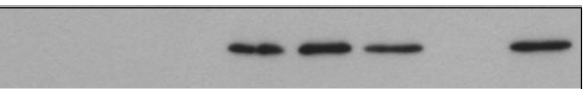

FDPS

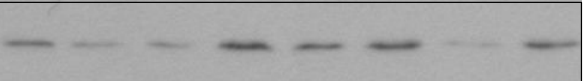

Ponseau S

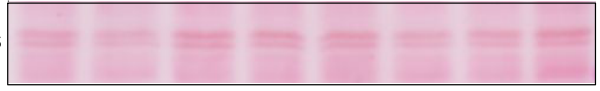

B

BALF
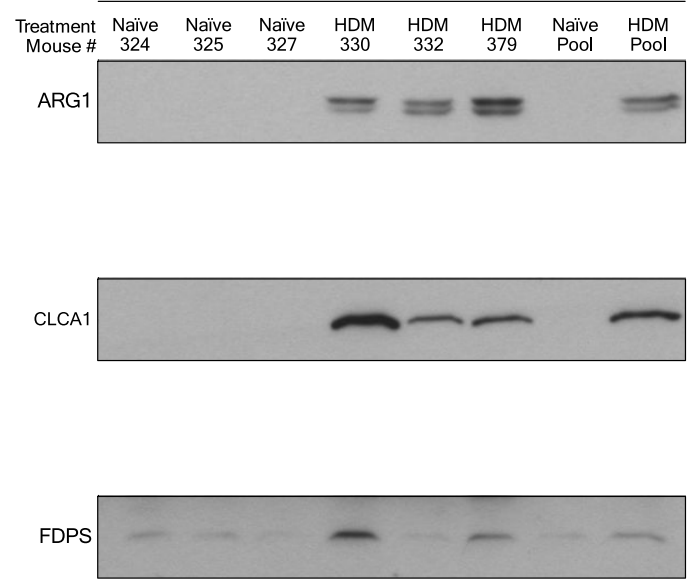

Ponseau S

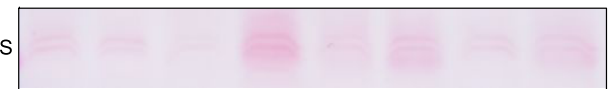

Western Blot

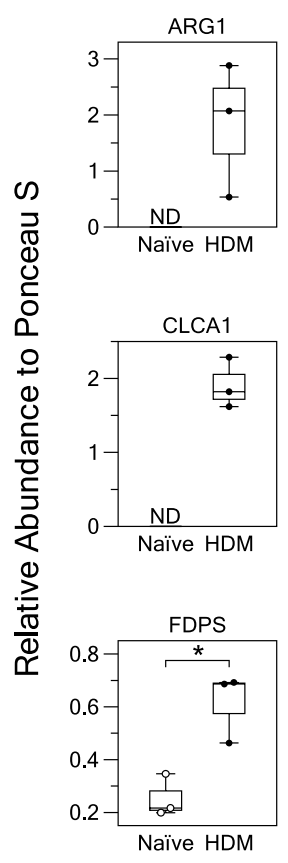

Western Blot

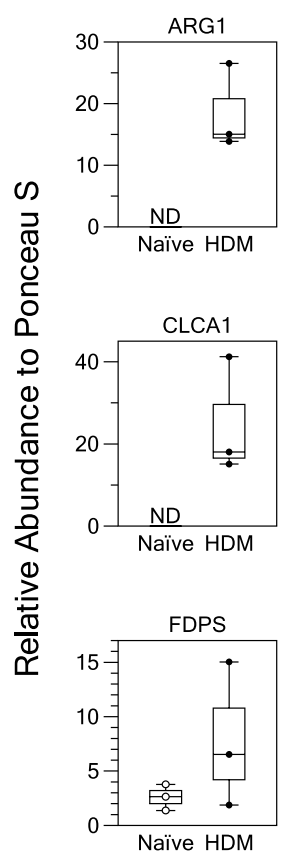

Proteomics

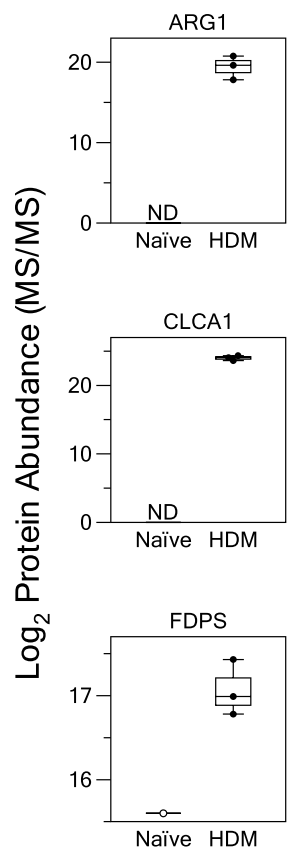

Proteomics

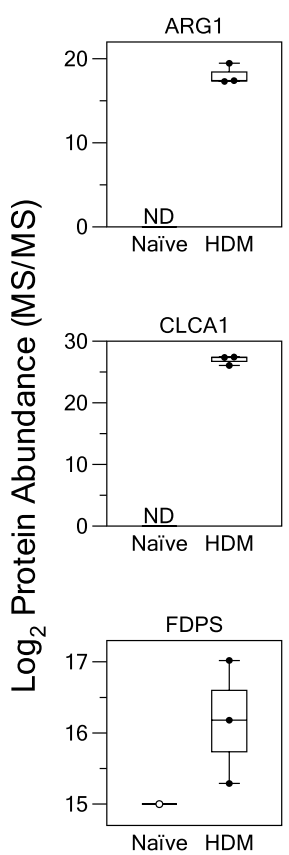

Figure 2. Western blot validation of lung tissue and BALF proteomics. (A, B) Images of the scanned chemiluminescent film for ARG1, CLC1, and FDPS. Protein bands were normalized to total protein loading (Ponceau S) for quantification. Abbreviations used: BALF: bronchial alveolar lavage fluid, * (Welch's unpaired $t$-test p.adj $\leq 0.05$ ), ND: not detected. Protein names: ARG1 (arginase-1), CLCA1 (calcium-activated chloride channel regulator 1), and FDPS (farnesyl pyrophosphate synthase).

Information, Figure S1A). Differential counting of BALF immune cells confirmed that HDM challenge triggered a significant accumulation of eosinophils and neutrophils that is also consistent with prior studies ${ }^{3}$ and mimics human asthma (Supporting Information, Figure S1B).

Lung Tissues and BALF Proteome Disparities. In total, our analysis of lung tissues and BALF samples in all mice yielded 2675 uniquely identifiable proteins (Protein IDs). On a per mouse basis, we obtained $1594 \pm 154$ protein IDs (mean $\pm \mathrm{SD}$ ) from lung tissues and $641 \pm 207$ protein IDs from BALF samples (Supporting Information, Figure S2A). To confirm that secreted proteins were enriched in the BALF samples, we characterized protein IDs using the Uniprot keyword, "secreted", which annotated $>75 \%$ of the proteins in the BALF. In contrast, only $25 \%$ of the protein ID's were characterized as secreted in the tissue lysate. To confirm that membrane-associated proteins are enriched in lung tissue samples, we classified proteins using the Uniprot annotated 
A

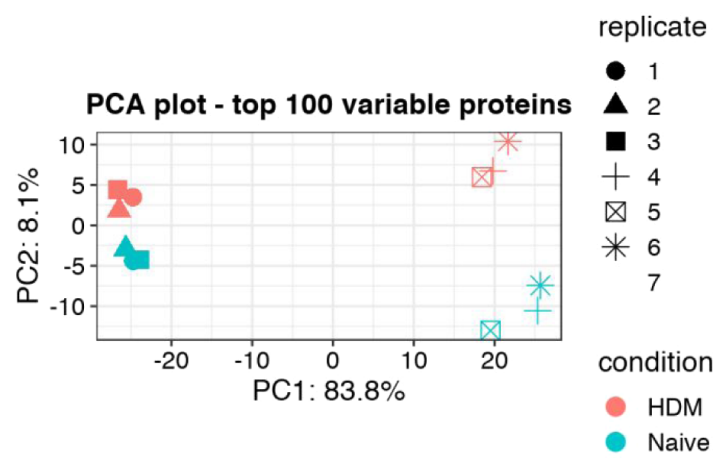

C

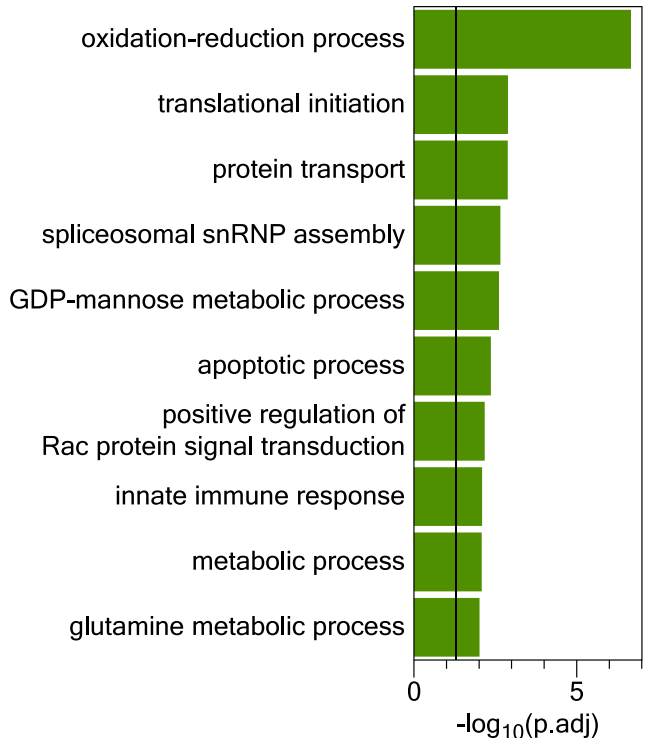

B

Combined Tissue-BALF (1246)
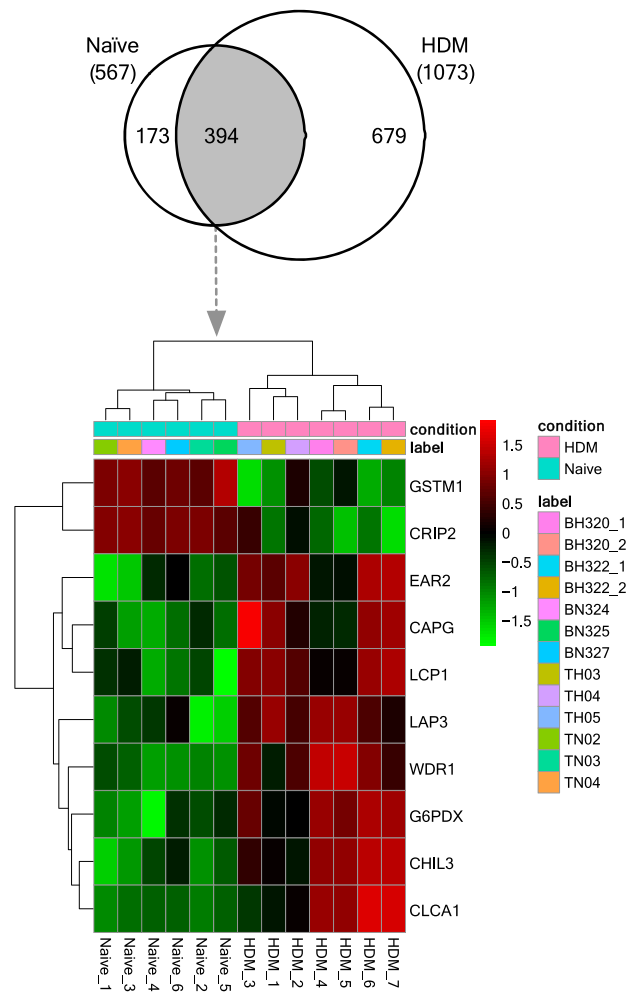

D

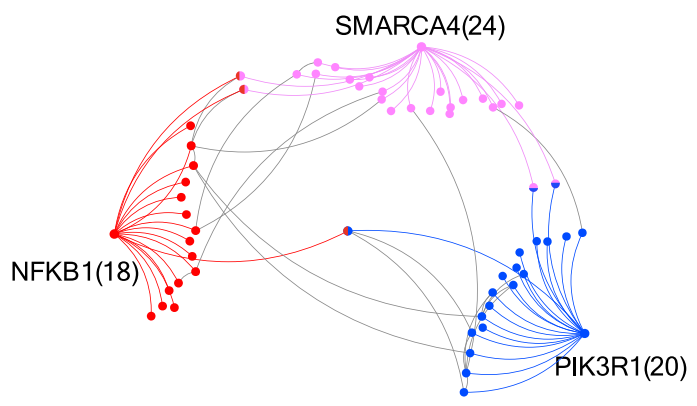

Figure 3. Combining tissue and BALF proteomes identified global processes in the lung of HDM-challenged animals. (A) PCA analysis highlights tissue samples and allergen driven differences in combined proteomes. (B) Venn diagram showing proteins common to naive and HDM proteomes and heatmap identifying differentially abundant proteins common to both proteomes. Red: high abundance, Green: low abundance. (C) Gene ontology biological processes enriched in the HDM proteome (p.adj $\leq 0.05$ ). The vertical line indicates a significance threshold. (D) Proteinprotein interaction network of the top three most interconnected protein-protein interaction nodes in the HDM proteome. Numbers beside each protein indicate the number of direct protein-protein interactions (first order). Abbreviations used: bronchial alveolar lavage fluid (BALF). Protein names: SMARCA4 (transcription activator BRG1), PIK3R1 (phosphatidylinositol 3-kinase regulatory subunit $\alpha$ ), and NF $\kappa \mathrm{B} 1$ (nuclear factor NFkappa-B p105 subunit).

keyword "transmembrane". This identified $69 \%$ of proteins in lung tissues and only $23 \%$ of BALF proteins.

To investigate if the proteomes of lung tissues and BALF from allergen-naive and HDM-exposed mice are distinct, we performed principal component analysis (PCA) on the top 500 most variable proteins across all samples. This cutoff was chosen arbitrarily to capture the high-intensity (and therefore highly variable) signals from our dataset. Our comparison matrix of all samples from allergen-naive and HDM-challenged mice using the top two principal components (PC) accounted for $\sim 80 \%$ of the variability between samples (Figure 1A). Lung tissue and BALF samples are separated along the first PC $(x-$ 
Table 1. Significantly Enriched or Depleted Proteins after HDM Challenge in a Combined Tissue-BALF Dataset ${ }^{a}$

\begin{tabular}{|c|c|c|c|c|c|}
\hline enriched/depleted in HDM & Uniprot ID & name & description & $\log _{2} \mathrm{FC}$ & p.adj \\
\hline enriched & $\mathrm{O} 35744$ & CHIL3 & chitinase-like protein 3 & 6.89 & 0.0018 \\
\hline enriched & P97425 & EAR2 & eosinophil cationic protein 2 & 2.69 & 0.0125 \\
\hline enriched & Q9D7Z6 & CLCA1 & calcium-activated chloride channel regulator 1 & 2.68 & 0.0133 \\
\hline enriched & Q9CPY7 & LAP3 & cytosol aminopeptidase & 2.44 & 0.00181 \\
\hline enriched & P24452 & CAPG & macrophage-capping protein & 2.39 & 0.0172 \\
\hline enriched & Q61233 & LCP1 & plastin-2 & 2.08 & 0.00692 \\
\hline enriched & O88342 & WDR1 & WD repeat-containing protein 1 & 1.26 & 0.014 \\
\hline depleted & Q9DCT8 & CRIP2 & cysteine-rich protein 2 & 2.33 & 0.00181 \\
\hline depleted & P10649 & GSTM1 & glutathione S-transferase $\mathrm{Mu} 1$ & 1.59 & 0.00679 \\
\hline
\end{tabular}

${ }^{a}$ Statistical threshold $\left(\log _{2} \mathrm{FC} \geq 1\right.$, p.adj $\left.\leq 0.05\right)$. Abbreviations: house dust mite (HDM), $\log _{2}$ fold change (LogFC).

axis), which accounts for approximately 59\% of the data variance. Lung tissue and BALF samples from naive and HDM-challenged mice were also distinct, with the second PC accounting for about $20 \%$ of data variance, with HDM effects seemingly much greater in BALF than in lung tissues (Figure $1 \mathrm{~A}$, expanded panel).

We next examined the distribution of proteins across all samples. A clustering heatmap ( $k$-means) shows that BALF and tissue clustered separately, as did naive and HDMchallenged tissue and BALF (Figure 1B). We also observed that the differences in lung tissue and BALF samples in allergen-naive and HDM-challenged mice were associated with the unique protein IDs in individual datasets. Approximately $72.7 \%$ of all protein IDs were unique to either lung tissue or BALF samples.

We next examined the distribution of Uniprot IDs in lung tissues and BALF and the effects of HDM challenge (Figure 1C). Venn diagrams show that HDM exposure increased the absolute number of proteins in lung tissue and BALF by 114 and $173 \%$, respectively (Supporting Information, File S3). The fraction of protein IDs common to BALF and lung tissues was $18 \%$ in allergen-naive mice, and this increased to $28 \%$ after HDM exposure. HDM exposure reduced the fraction of unique lung tissue Uniprot IDs from 75 to $63 \%$. The proportion of proteins that were unique to BALF was 8$10 \%$; this was not changed by HDM challenge.

We next examined patterns of protein abundance of the specific subset that were common to BALF and lung tissues from allergen-naive and HDM-challenged mice (Figure 1D). The dominating feature of the heatmap is that it discerns large clusters of proteins that are relatively more prominent in BALF or lung tissues, independent of the effects of HDM challenge. Thus, for these proteins common to lung and BALF, $k$-means clustering is consistent with PC analysis of all proteins (Figure 1A), which showed that data variability was chiefly the result of differences between lung tissues and BALF. Though the effects of HDM challenge within lung tissues or BALF are evident in this common protein subset, our analysis highlights the disparities between lung and BALF that likely affect the biological manifestation of HDM challenge.

Independent Validation of Proteins Selected from the Proteomic Analysis. To validate our proteomic data, immunoblotting was performed for three proteins in the same samples (Figure 2). Arginase-1 (ARG1), calcium-activated chloride channel regulator 1 (CLCA1), and farnesyl pyrophosphate synthase (FDPS) were selected based upon their enrichment by HDM exposure and the availability of reliable immunoblotting grade antibodies. Immunoblotting confirmed our proteomic data for ARG1 and CLCA1, as they were undetectable in allergen-näive samples from lung tissues and BALF, but prominent bands were evident for samples from allergen-challenged mice (Figure 2A,B). Immunoblotting analysis confirmed the presence of FDPS in all samples, with a significant enrichment of $270 \%$ (p.adj $\leq 0.05$ ) in the lung tissue after HDM challenge (Figure 2A,B). This is consistent with proteomic data for FDPS, which was detected in only one BALF and lung tissue sample from an allergen-naive mouse, while it was present and increased in all samples from HDMchallenged mice.

Combining Tissue-BALF Proteomes to Uncover Whole Lung Responses to HDM Challenge. To enable investigation of how HDM challenge affects protein interactions within the whole lung, we combined all protein IDs from BALF and tissue proteomes $(n=2675)$. To validate and reduce variability due to missing values, we filtered the dataset to include only those proteins that were detected in all biological replicates for each sample type and experimental condition. This strict criterion reduced the dataset to 1246 proteins for network and pathway analyses. The combined dataset of all tissue and BALF proteins included allergen-näive $(n=567)$ and HDM-challenged $(n=1073)$ proteins, both containing 394 proteins in common. Using this full dataset, we performed PCA to examine the effects of HDM challenge, and this showed that only $8.1 \%$ of the variation in protein abundance correlated with differences between naive and HDM-treated mice ( $Y$-axis, Figure $3 \mathrm{~A}$ ), whereas $83.8 \%$ of variation could be attributed to differences in protein abundance between lung tissues and BALF ( $X$-axis, Figure 3A).

Venn diagram mapping of protein IDs from the combined total lung tissue and BALF dataset showed that $68 \%$ of proteins were unique to allergen-naive (173) or HDMchallenged samples (679), and 32\% (394) were common to the treatment groups (Figure 3B). Proteins are listed in Supporting Information, File S3. Differential expression analysis of the 394 common proteins using LIMMA showed that two proteins were depleted, and eight proteins were enriched after HDM challenge (Table 1 and Figure 3B). To identify which biological processes were represented by these changes, we performed Gene Ontology analysis on a dataset that included the group of eight proteins enriched by HDM and the group of 679 proteins that were unique to samples from HDM-challenged mice (see Figure 3B). We identified 36 significantly enriched pathways, with the top five biological processes being: "oxidation-reduction process", "translational initiation", "protein transport", "spliceosomal snRNP assembly", and "GDP-mannose metabolic process" (Figure 3C). To 


\section{Tissue (Naïve vs HDM)}

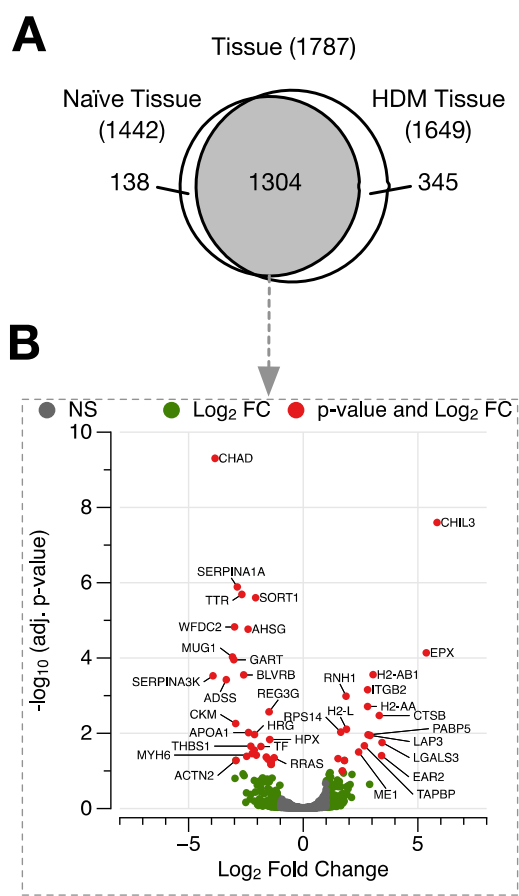

D

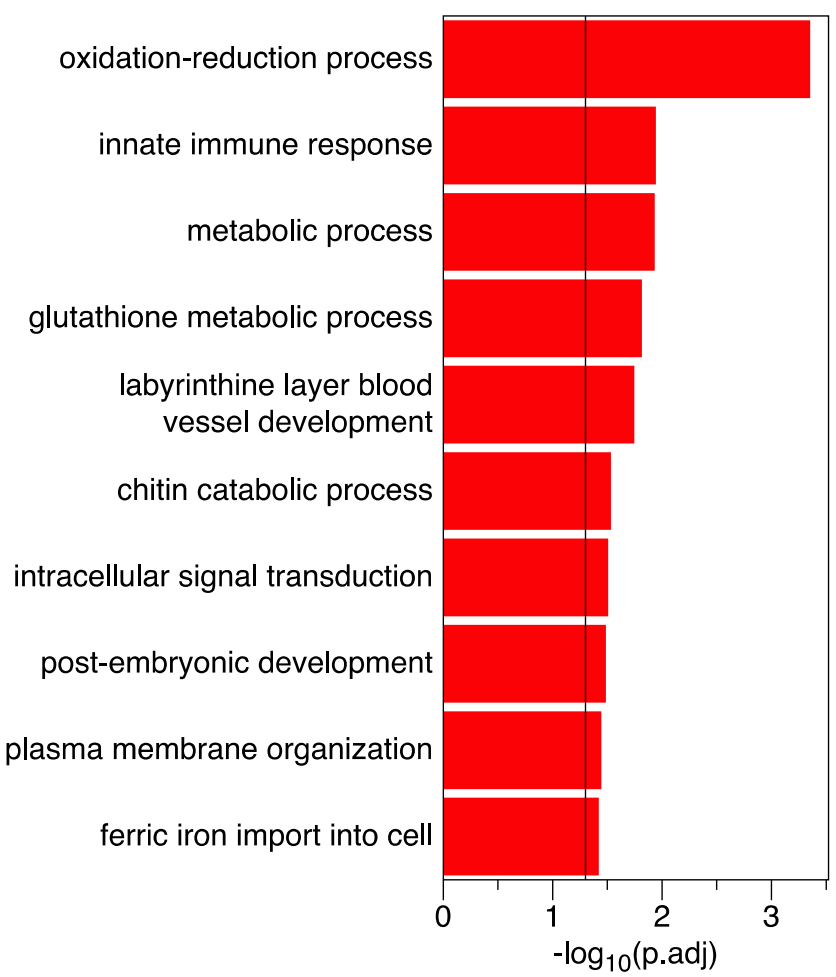

C Depleted in Tissue HDM

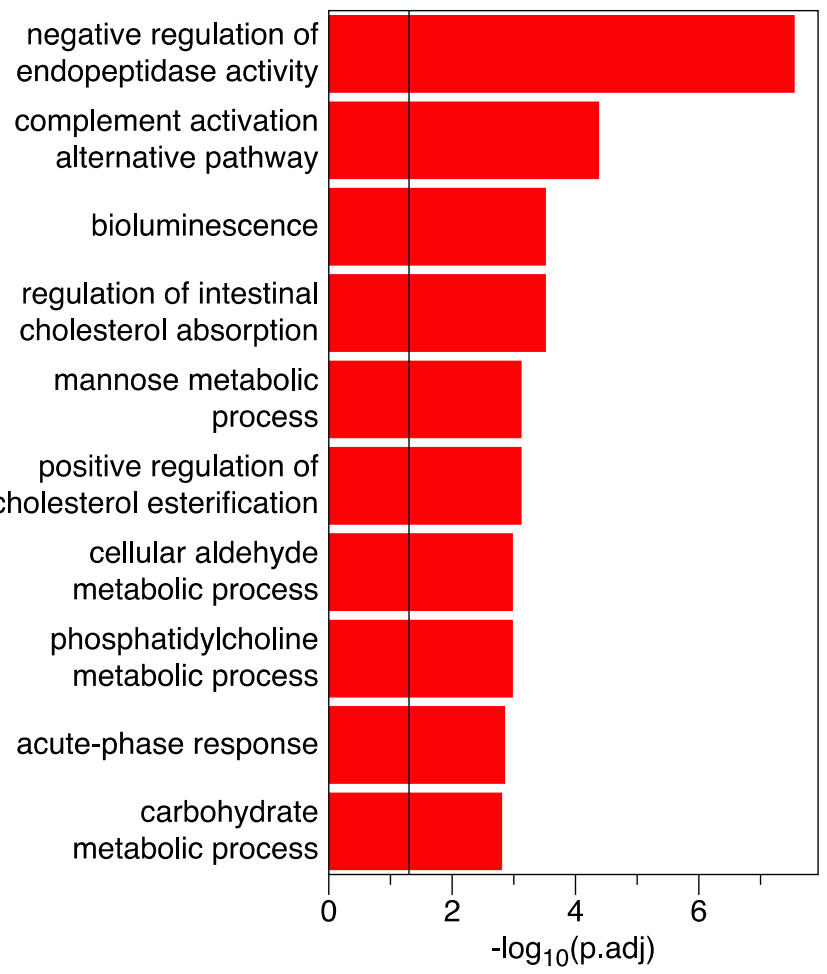

Figure 4. continued 
BALF (Naïve vs HDM)

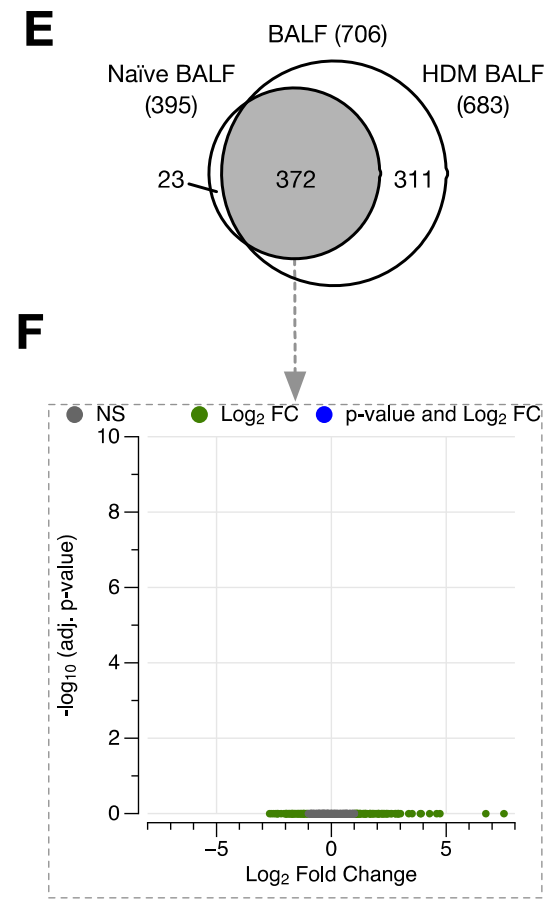

H

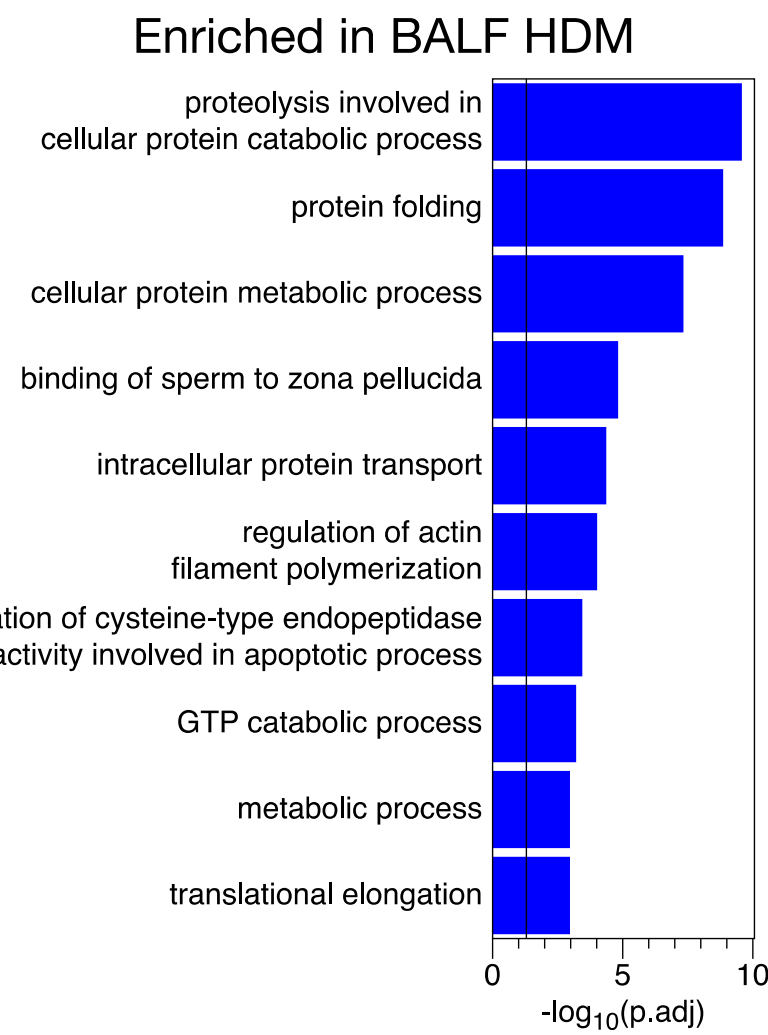

G

Depleted in BALF HDM

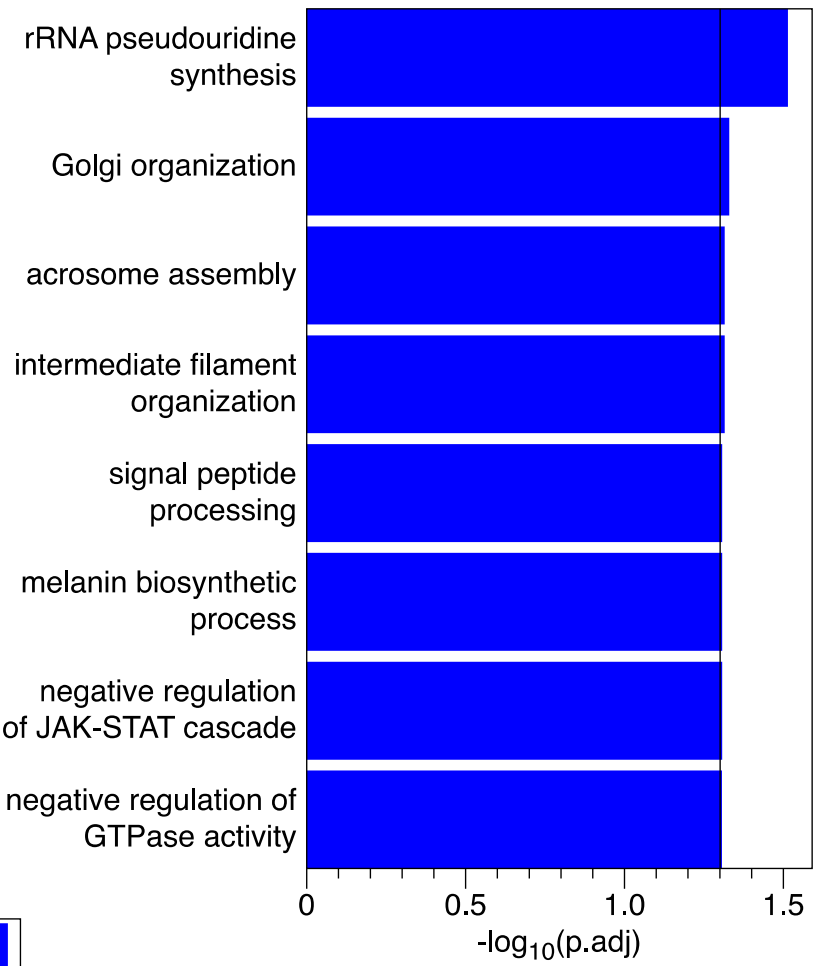

negative regulation

negative regulation of

GTPase activity

$-\log _{10}($ p.adj)

Figure 4. Individually analyzed HDM-influenced lung tissue or BALF proteomes do not recapitulate the biology of an integrated tissue-BALF proteome. (A) Venn diagram of the distribution of protein IDs between allergen-näive and HDM-exposed lung tissue datasets. (B) Volcano plot showing significantly altered proteins that were common to naive and HDM tissue proteomes. Red points are significant (p.adj < 0.05$)$ and have a $\log _{2} \mathrm{FC}>1$. Green points have a $\log _{2} \mathrm{FC}>1$ but are not significant. (C, D) Gene ontology analysis for enriched biological processes on differentially abundant and unique-to HDM proteins in lung tissue. (E) Venn diagram of the distribution of protein IDs between allergen-naive and HDMexposed BALF datasets. (F) Volcano plot showing significantly altered proteins that were common between naive and HDM BALF proteomes. No significantly altered proteins were detected. $(\mathrm{G}, \mathrm{H})$ Gene ontology analysis for enriched biological processes unique to HDM proteins in BALF. For brevity, only the top 10 nonredundant biological processes are shown. The vertical black line indicates significance $(p=0.05)$. 
A

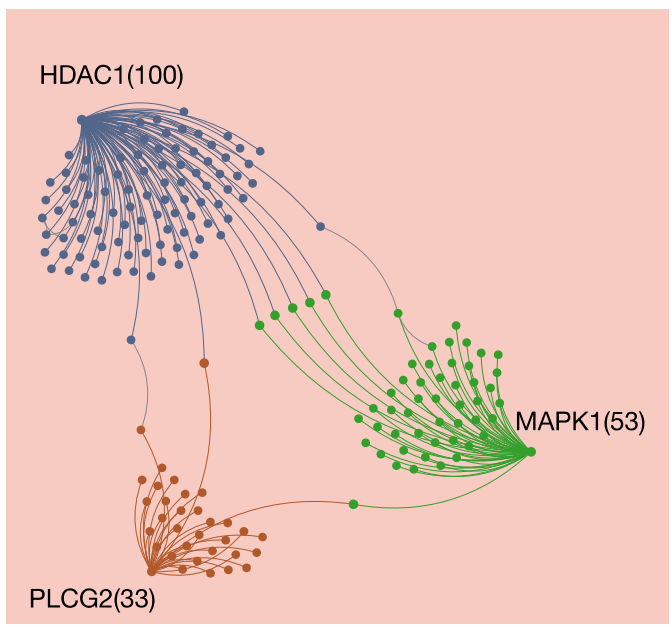

B

BALF

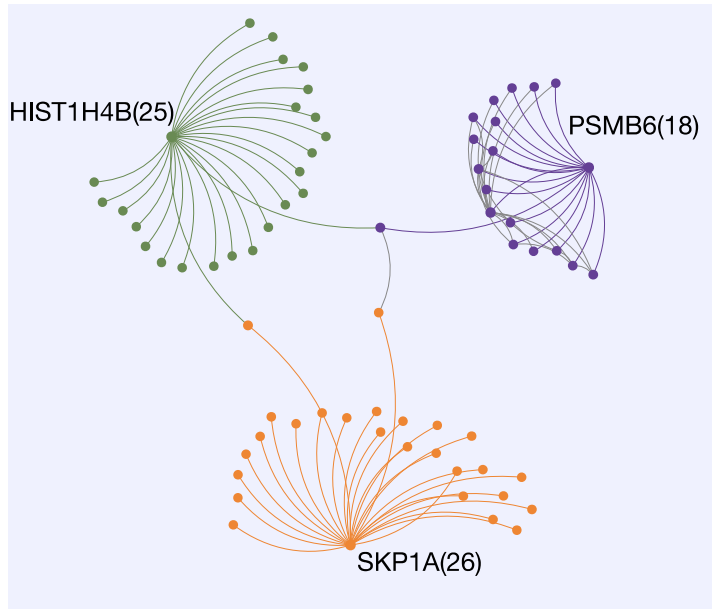

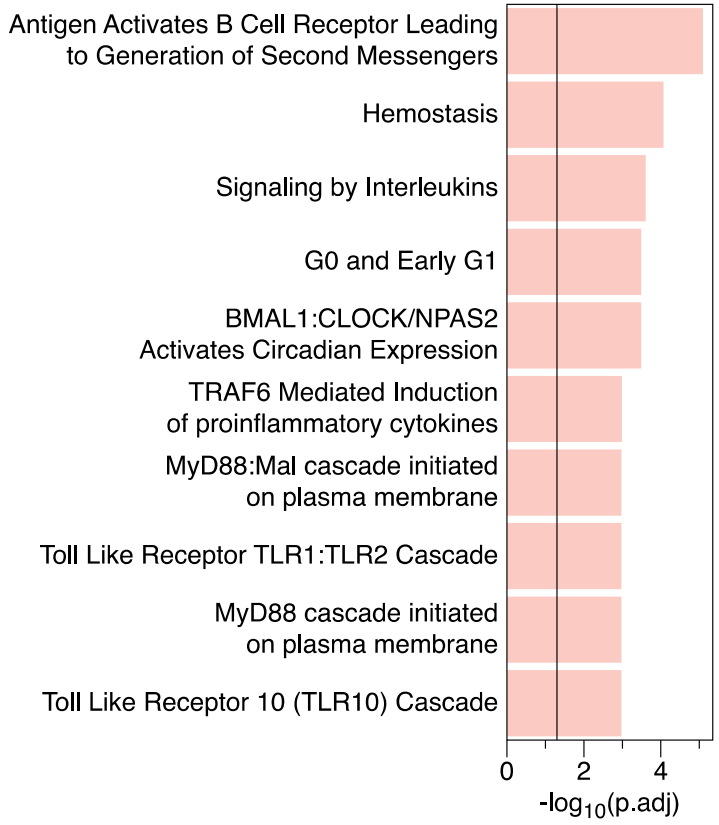

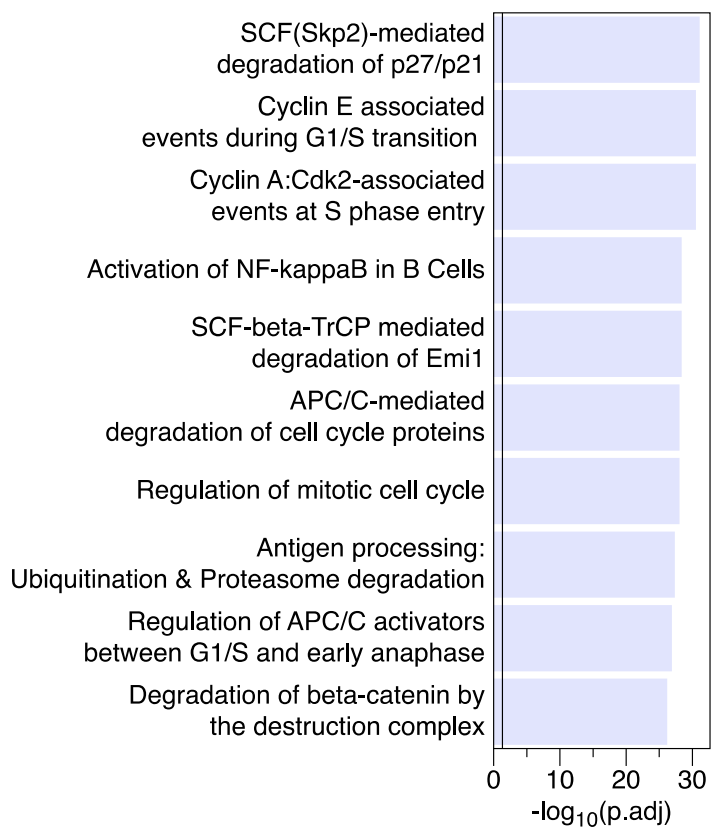

Figure 5. Most connected proteins in HDM-influenced lung tissue and BALF have distinct biological signatures. (A) Protein-protein interaction network of the top 3 most connected proteins in the HDM-influenced lung tissue proteome. (B) Protein-protein interaction network of the top 3 most connected proteins in the HDM-influenced BALF proteome. Biological pathway assessment from each network (Reactome) is shown on the right-hand side. Protein nodes and lines are colored to identify direct protein-protein interactions. Other parameters such as node size and line distance are used for illustrative purposes only. Numbers beside each protein indicate the number of direct protein-protein interactions (first order). Abbreviations used: house dust mite (HDM), bronchial alveolar lavage fluid (BALF). Protein names: HDAC1 (histone deacetylase 1), MAPK1 (mitogen-activated protein kinase 1), PLCG2 (1-phosphatidylinositol 4,5-bisphosphate phosphodiesterase $\gamma$-2), SKP1A (S-phase kinaseassociated protein 1A), HIST1H4B (H4-clustered histone 2), and PSMB6 (Proteasome subunit $\beta$ type-6).

complement this analysis, we performed a protein interaction analysis using NetworkAnalyst. The top three nodes, based on the number of connections with other proteins, included SMARCA4 (transcription activator BRG1), PIK3R1 (phosphatidylinositol 3-kinase regulatory subunit $\alpha$ ), and $\mathrm{NF} \kappa \mathrm{B} 1$ (nuclear factor NF-kappa-B p105 subunit) (Figure 3D). This set of networks was most significantly associated with "Interleukin-3,5 and GM-CSF signaling" (p.adj $=1.40 \times$
$10^{-8}$ ), forming a backbone for the whole lung response to HDM challenge.

Biological Significance of Proteome Changes Induced Specifically in Lung Tissues by Allergen Challenge. To assess lung tissue-specific effects of HDM challenge, we analyzed 1787 proteins that were unique to all lung tissue samples (Figure 4A). A large fraction of these proteins $(n=1304,75 \%)$ was common to allergen-naive and HDM-challenged mice, and differential abundance analysis 
Table 2. Network Complexity Increases When Combining the Lung Tissue and BALF Datasets That Are Uniquely Altered after HDM Exposure ${ }^{a}$

\begin{tabular}{|c|c|c|c|c|c|c|c|}
\hline \multirow{2}{*}{$\begin{array}{l}\text { dataset uniquely } \\
\text { altered by HDM }\end{array}$} & \multirow[b]{2}{*}{ Uniprot ID } & \multirow[b]{2}{*}{ name } & \multirow[b]{2}{*}{ description } & \multicolumn{2}{|c|}{ \# of interactions } & \multirow{2}{*}{$\begin{array}{l}\text { protein node enrichment in } \\
\text { the combined }(\%)\end{array}$} & \multirow{2}{*}{$\begin{array}{l}\text { number of first- and second-order } \\
\text { tissue-BALF interactions }\end{array}$} \\
\hline & & & & tissue & combined & & \\
\hline tissue & P83917 & CBX1 & $\begin{array}{l}\text { chromobox protein } \\
\text { homolog } 1\end{array}$ & 4 & 7 & 175.00 & 3 \\
\hline tissue & P61202 & COPS2 & $\begin{array}{l}\text { COP9 signalosome } \\
\text { complex subunit } 2\end{array}$ & 3 & 5 & 166.67 & 0 \\
\hline tissue & Q8CBW3 & $\mathrm{ABI1}$ & $\mathrm{Abl}$ interactor 1 & 5 & 8 & 160.00 & 0 \\
\hline tissue & O09106 & HDAC1 & histone deacetylase 1 & 23 & 34 & 147.83 & 7 \\
\hline tissue & P70429 & EVL & Ena/VASP-like protein & 5 & 7 & 140.00 & 3 \\
\hline
\end{tabular}

${ }^{a}$ The number of protein interactions (both first and second order) between the lung tissue and BALF datasets is shown (far right column).

uncovered 43 that were significantly changed after HDM exposure (28 depleted, 17 enriched) (Figure 4B). The proteins most significantly depleted by HDM challenge included SERPINA3K (serine protease inhibitor A3K), CHAD (chondroadherin), and ADSS (adenylosuccinate synthetase isozyme 2) (Supporting Information, File S3). We combined the 28 significantly depleted proteins with 138 proteins that were unique to allergen-naive lung tissue (therefore absent after HDM challenge) (Figure 4A). From this set of proteins that are uniquely depleted in the lung by HDM challenge, Gene Ontology analysis revealed that "negative regulation of endopeptidase activity" was the most significantly enriched biological process (Figure 4C).

Of the 17 proteins that were enriched ( $>\log _{2}$ fold change) by HDM challenge and that were common to allergen-naive lung tissue samples, CHIL3 (chitinase-like protein 3), EPX (eosinophil peroxidase), and LGALS3 (galectin-3) increased most significantly. We combined the 17 significantly enriched proteins with 345 proteins (Figure 4A) that were unique to HDM-challenged lung tissue samples. Using this set of 362 proteins uniquely enriched in lung tissues from HDMchallenged mice, Gene Ontology analysis revealed that "oxidation-reduction" regulation was the most significantly enriched process (Figure 4D).

To extend understanding of the differential abundance of the 362 proteins that we identified as being uniquely altered in allergen-exposed lung tissues, we used NetworkAnalyst to develop interactome maps that reveal interaction nodes and signaling networks for biological responses (Figure 5A). The top three interaction nodes for lung tissue were HDAC1 (histone deacetylase 1), MAPK1 (mitogen-activated protein kinase 1), and PLCG2 (1-phosphatidylinositol 4,5-bisphosphate phosphodiesterase $\gamma$-2), and these nodes generated an interactome involving multiple pathways. Using the Reactome database linked within NetworkAnalyst, the top five pathways were antigen-mediated B-cell receptor activation and secondary messenger generation; hemostasis; signaling by interleukins; proteins associated with G0 and Early G1; and activation of circadian expression through BMAL1:CLOCK/ NPAS2.

Biological Significance of Proteome Changes Induced Specifically in BALF by Allergen Challenge. To assess the effects of HDM challenge on the BALF-specific proteome, we analyzed 706 proteins that were unique to all BALF samples (Figure 4E). Approximately half $(n=372 ; 53 \%)$ of these protein IDs were common to BALF from allergennaive and HDM-challenged mice (Supporting Information, File S3). Further analysis for differential abundance in these common proteins did not reveal any that were significantly enriched or depleted by allergen challenge (Figure 4F). Therefore, we performed Reactome pathway analysis (linked within NetworkAnalyst) using the 23 proteins that had only been identified in allergen-naive BALF samples (Figure 4E). This discriminated 10 biological processes that are uniquely depleted by HDM challenge in BALF, with "rRNA pseudouridine synthesis" being the most significantly affected (Figure 4G). To determine pathways in BALF that were enriched by HDM challenge, we also performed Gene Otology analysis in InnateDB using the 311 proteins that were unique to BALF from HDM-challenged mice (Figure 4E). This revealed top 10 biological processes uniquely enriched by the challenge to inhaled allergen in BALF, and this included "proteolysis involved in cellular protein catabolic process(es)" as the most significantly enriched (Figure $4 \mathrm{H}$ ).

To extend understanding of the 311 proteins that are uniquely altered in allergen-exposed BALF, we developed interactome maps to identify primary interaction nodes and signaling networks (Figure 5B). Using the Reactome database in NetworkAnalyst, the top three protein-protein interaction (PPI) nodes were SKP1A (S-phase kinase-associated protein 1A), HIST1H4B (H4-clustered histone 2), and PSMB6 (Proteasome subunit $\beta$ type-6). This PPI network included diverse pathways, with the top 5 pathways being the SCF(Skp2)-mediated degradation of p27/p21, Cyclin Eassociated events during G1/S transition, Cyclin A:Cdk2associated events at $S$-phase entry, activation of NF- $\kappa$ B in B cells, and SCF- $\beta$-TrCP-mediated degradation of Emil.

Predicting Interactions between Lung Tissues and BALF. To broaden the scope of our study, we interrogated protein interactions across biological compartments using the lung tissue and BALF samples collected from each mouse. To uncover points of integration of biological activities in lung tissue and the surrounding extracellular space in response to allergen challenge, we integrated the 362 proteins that were uniquely enriched by HDM challenge in lung tissue (see Figures $4 A, B$ and $5 \mathrm{~A}$ ) with the 311 proteins that were uniquely enriched in BALF (see Figures 4E,F and 5B). Importantly, as this integration directly compares the interactions between the lung tissue and BALF, we excluded the 49 proteins that are shared between the two datasets, resulting in 311 and 262 proteins found exclusively in the allergen-exposed lung tissue and BALF datasets, respectively. We next identified protein interaction nodes in the combined dataset using NetworkAnalyst, enhancing the rigor of our analyses by filtering the results to only include protein nodes with five or more firstorder interactions. From the 37 proteins that met this criterion 
Table 3. Network Complexity Does Not Change When Combining the BALF and Lung Tissue Datasets That Are Uniquely Altered after HDM Exposure ${ }^{a}$

\begin{tabular}{|c|c|c|c|c|c|c|c|}
\hline \multirow{2}{*}{$\begin{array}{l}\text { dataset uniquely } \\
\text { altered by HDM }\end{array}$} & \multirow{2}{*}{$\begin{array}{l}\text { Uniprot } \\
\text { ID }\end{array}$} & \multirow[b]{2}{*}{ name } & \multirow[b]{2}{*}{ description } & \multicolumn{2}{|c|}{ \# of interactions } & \multirow{2}{*}{$\begin{array}{l}\text { protein node enrichment } \\
\text { in the combined (\%) }\end{array}$} & \multirow{2}{*}{$\begin{array}{l}\text { number of first- and second- } \\
\text { order BALF-tissue interactions }\end{array}$} \\
\hline & & & & BALF & combined & & \\
\hline BALF & Q62261 & SPTBN1 & $\begin{array}{l}\text { spectrin } \beta \text { chain, non- } \\
\text { erythrocytic } 1\end{array}$ & 10 & 10 & 100.00 & 7 \\
\hline BALF & P62908 & RPS3 & $40 S$ ribosomal protein $S 3$ & 7 & 7 & 100.00 & 1 \\
\hline BALF & Q99020 & HNRNPAB & $\begin{array}{l}\text { heterogeneous nuclear } \\
\text { ribonucleoprotein } \mathrm{A} / \mathrm{B}\end{array}$ & 6 & 6 & 100.00 & 2 \\
\hline BALF & Q9Z2U1 & PSMA5 & $\begin{array}{l}\text { proteasome subunit } \alpha \\
\text { type-5 }\end{array}$ & 5 & 5 & 100.00 & 2 \\
\hline BALF & Q9R1P3 & PSMB2 & $\begin{array}{l}\text { proteasome subunit } \beta \\
\text { type- } 2\end{array}$ & 5 & 5 & 100.00 & 3 \\
\hline
\end{tabular}

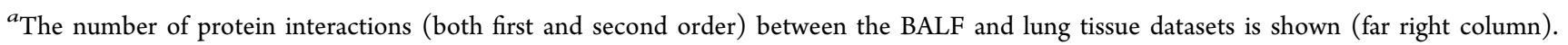

(12 from lung tissue, 25 from BALF), we selected the top 5 proteins from both the lung tissue and BALF datasets that become enriched in the combined dataset. We found that the integration of BALF proteins with lung tissue-specific proteins resulted in a significant enrichment, $142.1 \pm 19.1 \%$ (range of $122-185 \%$ ), of first-order interactions for nodes that emerged from lung-derived seed proteins (the proteins that start the growth of a network) (Table 2). In contrast, combining lung tissue proteins with those from BALF did not affect the number of interactions for seed protein nodes that originated from BALF (Table 3).

To uncover points of crosstalk between lung tissues and BALF proteins, we built a combined interaction network using NetworkAnalyst (Figure 6). Once we removed the proteins that are common between the two datasets, we calculated the number of interactions each protein has within from lung tissue $(n=311)$ and BALF $(n=262)$ datasets. We then extracted the top five proteins from the lung tissue that increased as a result of combining with the BALF dataset (Table 2). These included HDAC1, CBX1 (chromobox protein homolog 1), EVL (Ena/VASP-like protein), ABI1 (Abl interactor 1), and COPS2 (COP9 signalosome complex subunit 2). Since combining these datasets did not enrich the number of interactions from each protein node derived from BALF, for interactome mapping we included the top five seed proteins that maintained interactions when combined with lung tissue proteins. These included SPTBN1 (Spectrin $\beta$ chain, non-erythrocytic 1), RPS3 (40S ribosomal protein S3), HNRNPAB (heterogeneous nuclear ribonucleoprotein A/B), PSMA5 (proteasome subunit $\alpha$ type-5), and PSMA2 (proteasome subunit $\alpha$ type-2) (Table 3).

A unique aspect of our analytical approach is that it uncovered six predicted integration proteins between key pathway nodes in lung tissues and BALF. As shown in Figure 6, these included NANOG (homeobox protein NANOG), POU5F1 (POU domain, class 5, transcription factor 1), TFCP2I1 (transcription factor CP2-like protein 1), SMAD4 (mothers against decapentaplegic homolog 4), TAL1 (T-cell acute lymphocytic leukemia protein 1 homolog), and YWHAZ (14-3-3 protein zeta/delta), and each protein had two to four interactions spanning lung tissues and BALF. Among lungderived seed proteins, HDAC1 interacted with seven predicted integrating proteins and thus associated with all of the BALForigin protein nodes. Neither COPS2 nor ABI1 signaling nodes from lung tissue were associated with any of the predicted integration proteins from the BALF dataset, although indirect linkage of $\mathrm{ABI} 1$ via EVL and $\mathrm{CBX} 1$ is apparent.
Among BALF-derived seed nodes, SPTBN1 and HNRNPAB exhibited six and two links, respectively, to predicted integration proteins, thus creating interactions to HDAC1 and EVL in lung tissue. As well, PSMA2 and PSMA5 from BALF projected three interactions to lung tissue nodes (HDAC1 and EVL). Similarly, SPTBN1 in BALF predicted direct linkages to SMAD4, TAL1, and YWHAZ along with indirect linkages between EVL, HDAC1, and CBX1 (Figure $6 \mathrm{~A})$. Together, these results reveal that combining proteins in a manner that allows identification of the specific biological compartment from which they were derived enables the critical discrimination of pathways that link the allergic response in lung tissues with that in the surrounding extracellular space.

The impact of combining data for proteins that are uniquely enriched by HDM challenge in matched BALF and lung tissue samples is also evident in the divergence of predicted biological processes that emerges, compared to those based on lung tissue or BALF datasets alone. Figure $6 \mathrm{~B}, \mathrm{C}$ lists the top 10 biological processes predicted using the Reactome database in NetworkAnalyst for lung tissues and BALF, using the top five seed proteins derived from each, but as influenced by the combined dataset. Prominent lung tissue processes are associated with immune system regulation (innate and adaptive), including $\mathrm{T}$-cell signaling and hemostasis, as well as tissue remodeling ( $\mathrm{ROBO}$ receptors), and cell division. BALF seed proteins are linked to processes associated with cell stasis (apoptosis, protein turnover, and DNA damage control pathways, including ornithine decarboxylase), as well as antigen presentation. Figure $6 \mathrm{D}$ represents the predicted biological processes using all ten seed protein nodes from BALF and lung tissue combined. The list of biological processes reflects a strong dominance by lung tissue-linked processes, in particular immune responses and the regulation of mitosis and cell division. Of note, two areas that are not evident in lung tissues and BALF alone but are known components of allergic asthma pathobiology were revealed by the integration of datasets, including B-cell receptor signaling and inflammation, featuring NF- $\kappa \mathrm{B}$ activation, pathways induced by $\mathrm{Wnt}$, and degradation of $\beta$-catenin.

\section{DISCUSSION}

Murine models of allergic airway inflammation employ inhaled aeroallergen such as HDM to support preclinical and discovery research. We employed a long-established model of allergic asthma that is known to induce airway hyper-responsiveness, airway inflammation, and airway remodeling. ${ }^{1,3,7}$ Asthma pathobiology involves interaction between recruited inflam- 
A

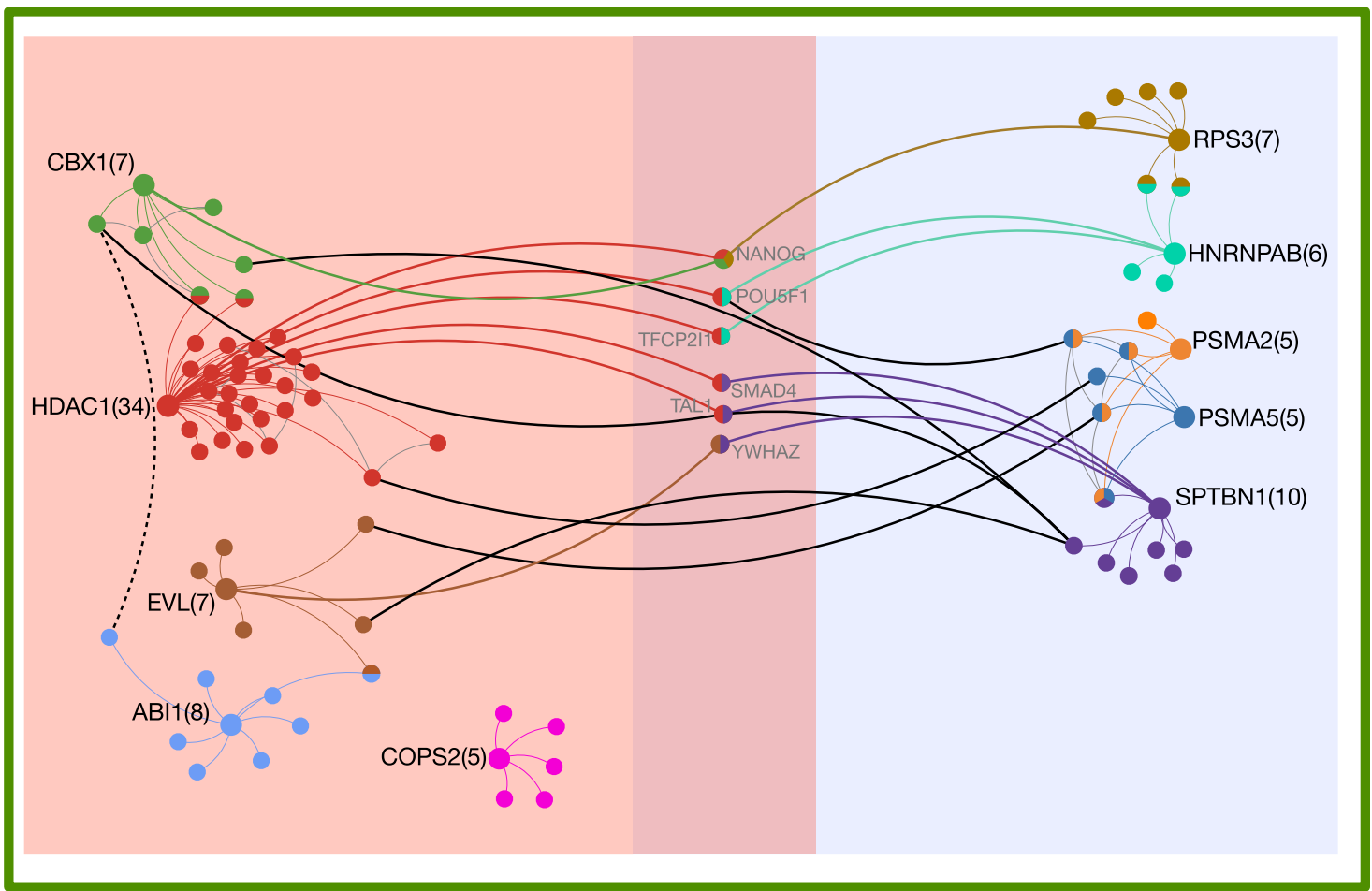

B

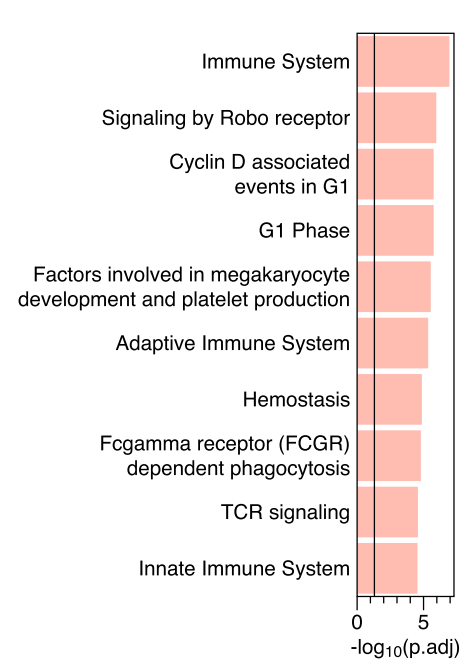

C

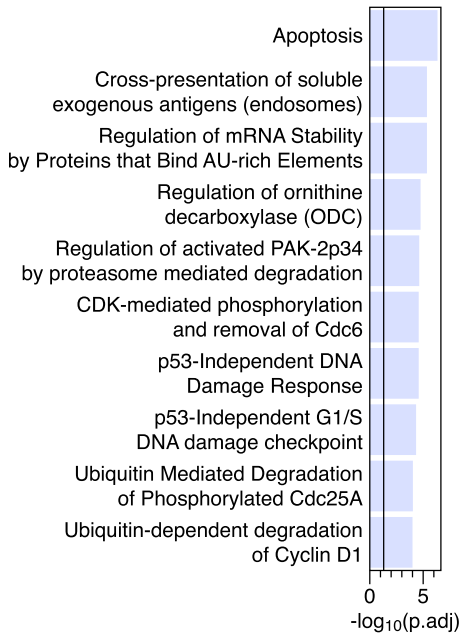

D Combined

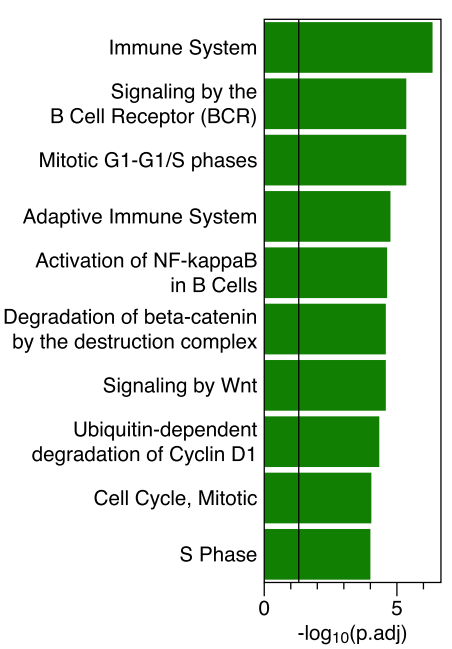

Figure 6. Integrating proteomes enriches protein-protein interactions and reveals points of connection between lung and BALF proteomes. (A) Protein-protein interaction network of the top 5 most connected proteins in the HDM-influenced lung tissue and BALF proteomes. Lung tissue nodes (light red) are shown on the left. BALF nodes (light blue) are shown on the right. Protein nodes that are directly involved (first order) in lung tissue-BALF crosstalk are shown in the center shaded region (purple) and are labeled in gray. The combined dataset is representative of all protein nodes within the green box. (B-D) Biological processes derived from the Reactome dataset are color-coordinated to their respective networks and each includes the six proteins in common (purple). In panel (A), circles represent individual proteins, while protein-protein interactions are shown as lines. Line color that matches seed nodes indicates direct protein interactions with seed nodes. Circles with split colors indicate shared first-order interactions between multiple seed nodes. Black lines indicate second-order (indirect) inter-dataset (lung tissue-BALF) crosstalk. Broken lines indicate intra-dataset node interactions (second order). The number of direct protein-protein interactions (first order) for each seed node is shown in parentheses. Other parameters such as node size, node distribution, and line distance are used for illustrative purposes only. Abbreviations used: bronchial alveolar lavage fluid (BALF). Protein names: HDAC1 (histone deacetylase 1), CBX1 (chromobox protein homolog 1), EVL (Ena/VASP-like protein), ABI1 (Abl interactor 1), COPS2 (COP9 signalosome complex subunit 2), SPTBN1 (spectrin $\beta$ chain, non-erythrocytic 1), RPS3 (40S ribosomal protein S3), HNRNPAB (heterogeneous nuclear ribonucleoprotein A/B), PSMA5 (proteasome subunit $\alpha$ type-5), PSMA2 (proteasome subunit $\alpha$ type-2), NANOG (homeobox protein NANOG), POU5F1 (POU domain, class 5 , transcription factor 1), TFCP2I1 (transcription factor CP2-like protein 1), SMAD4 (mothers against decapentaplegic homolog 4), TAL1 (T-cell acute lymphocytic leukemia protein 1 homolog), and YWHAZ (14-3-3 protein zeta/delta). 
matory cells and lung structural cells. In this study, we used label-free proteomics and multivariate bioinformatics to describe and compare the molecular interactome in lung tissues and BALF from HDM-challenged mice. We used matched samples from individual mice for this process and in so doing have been able to discriminate responses in tissue and extracellular spaces. Using in silico reintegration of datasets, we predict the interactions between lung tissues and the BALF. We demonstrate that the proteomes of lung tissues and BALF are remarkably different, and this is further enhanced by disparate effects resulting after HDM challenge, highlighting inherent biological diversity in these samples. Examining protein-protein interactions between lung tissues and BALF reveals points of crosstalk between lung tissues and extracellular proteins after HDM exposure. Our study reveals the scope and limits of biological insight that can be obtained from lung tissue or BALF sample proteins alone and offers the potential to interrogate network interactions between the lung tissue and extracellular airway space during allergen challenge.

Confirming a B-Cell Signaling Immune Signature in BALF and Lung Tissues. In the current study, we confirmed that HDM challenge led to predominant eosinophil and neutrophil infiltration of BALF, and we found B-cell receptor signaling to be a key component of, and crosstalk between, lung tissues and BALF in allergen-exposed mice (Figures 5A and $6 \mathrm{D})$. The immune signature evident in lung tissues and BALF after HDM challenge is predominantly of a Th2polarized phenotype, in part dependent on endotoxin levels in HDM. $^{7,8}$ Murine B cells are antigen-presenting cells for HDM antigens, and B-cell depletion profoundly reduces allergic responses in the lung due to their interactions with resident lung cells and inflammatory cells that infiltrate the lung and airways. ${ }^{9}$ Importantly, memory B-cell populations remain in the lung tissue and epithelial cell layers well after HDM sensitization, forming a localized population that can quickly become activated by further allergen challenge. ${ }^{10}$ This pattern of a localized memory B-cell population contributes to inflammatory responses that associate with airway remodeling in asthmatic patients. ${ }^{11}$ As a biological validation of our proteomic investigation, our chief findings reveal a B-cell signaling signature after repeated allergen challenge. Moreover, our network analysis of integrated lung tissue (collected post lavage) and BALF proteomes reveals multiple levels for crosstalk between the lung tissue and the extracellular microenvironment. In addition to the immunoblot validation of proteins in lung tissues and BALF that we provide, these observations provide evidence of the reliability of our proteome measurement and analysis protocols and further support an important role for B cells in coordination of biological responses in lung tissues and BALF.

Protein Nodes That Link Lung Tissues and BALF. In lung tissue samples, we detected markedly increased HDAC1 (histone deacetylase 1) after allergen exposure, and it was a seed protein for a major interaction node. Moreover, via all six of the proteins we predicted to integrate lung tissue and BALF responses (Figure 6), HDAC1 appears to interact with all of the BALF protein nodes in HDM-challenge mice. This suggests a fundamental role for HDAC1 in allergic pathophysiology, which is consistent with its primary role, in conjunction with histone acetyltransferase (HAT), to modulate chromatin accessibility for transcription. Our finding is consistent with evidence from ovalbumin-sensitized and challenged mice, in which HDAC1 abundance and activity is increased. $^{12}$ Skewed HDAC and HAT activity has been documented in both asthmatic and chronic obstructive pulmonary disease (COPD) patients, ${ }^{13,14}$ and HDAC1 activity negatively correlates with disease severity and IL-8 expression. ${ }^{14}$ This link with inflammation is due, in part, to transcriptional control of NF- $\kappa \mathrm{B}$ signaling and its transcriptional activation of pro-inflammatory genes and antimicrobial peptides. ${ }^{15-17}$ Together, our study reveals the complexity of HDAC regulation and suggests that modification of transcriptional events leads to physiological and inflammatory changes that span beyond a single biological compartment.

CBX1 abundance was increased in lung tissues after allergen exposure but exhibited limited interactions with BALF proteins. CBX1 (chromobox protein homolog 1), a heterochromatin binding protein associated with transcriptional regulation of cell proliferation, also interacts with nuclear lamin (LMNB1) and EMSY (BRCA2-interacting transcriptional repressor). ${ }^{18,19}$ EMSY is an interferon- $\alpha / \beta$ gene regulator, $^{20}$ and a genome-wide association study recently identified EMSY (C11orf30-LRRC32) to be significantly associated with moderate-to-severe asthma. ${ }^{21}$ Similar to CBX1, ABI1 increased significantly in lung tissues after HDM challenge, but network analysis did not reveal interactions with protein nodes in BALF. ABI1 associates with N-WASP to regulate filamentous actin dynamics, as well as acetylcholine-mediated contraction of the airway smooth muscle. ${ }^{22} \mathrm{ABI1}$ is regulated by the tyrosine kinase $\mathrm{ABL},{ }^{22}$ which is increased in lungs of asthmatic donors and allergenchallenged mice. ${ }^{23}$ Moreover, conditional ABL knockout mice are refractory to allergen challenge-associated increased airway resistance, airway smooth muscle mass, and tracheal contractility. ${ }^{23}$ Collectively, our finding of lung tissue-specific induction of CBX1 and ABI1 is consistent with the presence of inherent biological pathways that are primary drivers of fibroproliferative changes that are associated with airway remodeling $^{3}$ and airway hyper-responsiveness.

In BALF samples from allergen-challenged mice, SPTBN1 (Spectrin $\beta$ chain, non-erythrocytic 1 ; also known as ELF) was increased in abundance, and our network analyses revealed linkage to HDAC1 in tissues via SMAD4 and TAL1. Spectrin is associated with cytoskeletal maintenance with ankyrin and anion exchanger 1 (AE1) to stabilize cortical actin linkage to the cell membrane. ${ }^{24}$ Spectrin repeat and $\mathrm{Ph}$ domains of SPTBN1 bind cell membrane phospholipids, thus is positioned to respond to, and effect, interactions between cells in complex biological systems. ${ }^{25,26}$ SPTBN1 also has a critical role in TGF$\beta$-induced gene transcription, as SMAD3/4 becomes mislocalized and dysfunctional in the SPTBN1 knockout mouse. ${ }^{27}$ This is critical as TGF- $\beta$-induced SMAD3/4 signaling can induce human airway smooth muscle cell shortening and hyper-responsiveness and is a primary extracellular driver of asthma pathobiology. ${ }^{28}$ Based on its key role in mediating cytoskeletal structure and TGF- $\beta$ signaling, our finding that SPTBN1 is induced in BALF likely reflects its role in cell-cell interactions, including paracrine signaling systems.

In BALF from allergen-exposed mice, we detected increased PSMA2 and PSMA5 (proteasome subunit $\alpha-2$ and -5). These proteins were distinct in that interactions with lung tissue protein nodes were limited to single links with HDAC1 and EVL, suggesting that their primary roles lie within the BALF compartment. PSMA2 and PSMA5 maintain structure and function of the 20S proteasomal complex and have increased activity in BALF from acute respiratory distress patients. ${ }^{29,30}$ 
Intratracheal instillation of a proteasome inhibitor reduces NF$\kappa \mathrm{B}$ signaling and eosinophil number in the BALF from OVAexposed rats. ${ }^{31}$ A definitive role for proteasomal complexes in BALF after allergen challenge is unclear, but they have been implicated in diverse processes such as the formation of exogenous peptide antigens for immune cells, degradation of oxidatively damaged proteins, and activation of precursor proteins. ${ }^{32}$ We identified PSMA2 and PSMA5 as a significant node in BALF that is relatively disconnected from lung tissue protein nodes, suggesting that their principal role lies in the regulation of inflammation, involving inflammatory cells, and perhaps their interaction with structural cells of the lung.

Proteins That Mediate Lung Tissue-BALF Crosstalk in Allergen-Exposed Mice. We identified remarkable differences in the protein profile between lung tissues and BALF, even more so than the proteome changes that develop in each sample type after allergen challenge. This highlights a need to consider a role for protein interactions between lung tissues and BALF after allergen challenge to fully interrogate lung system responses to allergic insult. To meet this need mandated use of a unique design, in which we collected lung tissue and BALF samples from each mouse, performed proteomic analysis in each sample, and then recombined datasets to predict how the most significant protein nodes in lung tissues and BALF interact. Protein interaction network analysis identified six proteins, NANOG, POU5F1, TFCP2I1, SMAD4, TAL1, and YWHAZ, that are present in both lung tissue and BALF datasets, and that could be pivotal mediators of coordinated responses involving both biological compartments.

NANOG (homeobox protein NANOG) and POU5F1 (POU domain, class 5, transcription factor 1; also known as OCT4) are transcription factors that can function in concert to regulate several genes, including EPHA, FGF2, SMAD1, and SKIL, that are of relevance to lung diseases. These targets regulate cell matrix production and cell motility (EPHA), ${ }^{33}$ induce airway smooth muscle hyperplasia (FGF2), ${ }^{34}$ and provide transcriptional control of TGF- $\beta$, either directly by receptor-activated SMAD $1,{ }^{35}$ and indirectly, by the inhibitory protein, SKIL (also called SnoN). ${ }^{36}$ SKIL, which is part of a family of proteins, including SKI and Sno that repress transcription of TGF- $\beta$ response genes, and interacts with SMAD3 and SMAD4. Interestingly, as noted above, network analysis identified SMAD4, which binds TGF- $\beta$ induced RSmads to form transcriptional activator complexes, and TAL1, a hematopoietic transcription factor, as regulators of coordinated responses in BALF and lung tissues. SKI, SKIL, and other TGF- $\beta$ signaling inhibitors regulate cell responses that are dependent on SMAD4 and TAL1. ${ }^{37,38}$ TGF- $\beta$ is a central driver of asthma pathobiology, and its elevated levels in lung tissues and BALF are associated with diverse effects. Highlighting this, although in mice neutralizing antibodies for TGF- $\beta$ have little effect on HDM challenge-induced lung dysfunction and airway smooth muscle thickening, they do alter the nature and degree of airway inflammation. ${ }^{39}$ Overall, our analysis reveals that coordination of responses in BALF and lung tissue compartments involves considerable transcriptional regulation, in particular related to the broad biological effects of TGF- $\beta$ on tissue repair and remodeling, inflammation, immunity, and hematopoietic cell maturation and activation.

Our intercompartment network analysis also points to the airway epithelium and a key zone for lung tissue-BALF crosstalk, which is consistent with the fact that epithelial barrier function disruption and mucus hypersecretion are hallmark features of the response to repeated allergen inhalation. We identified the transcription factor, TFCP2I1 (transcription factor CP2-like protein 1; also known as Crtr-1) as a novel co-ordinating protein node. Notably, it has diverse roles in epithelial cell maturation and modulating $\beta$-catenin signaling. ${ }^{40,41}$ To our knowledge, a role for TFCP2I1 in allergic lung pathobiology has not been reported, but in kidneys and salivary glands, it is a crucial regulator of epithelial cell maturation. ${ }^{40}$ There is evidence from lung cancer studies that TFCP2I1 directly interacts with YWHAZ and protects $\beta$ catenin from degradation. ${ }^{42}$ YWHAZ, also called $14-3-3 \zeta$, is another protein we predict to be a coordinator of lung tissueBALF biological responses. It is a binding partner with numerous cytoplasmic and nuclear proteins and modulates signaling associated with apoptosis and cell cycle progression. $^{43,44}$ Furthermore, 14-3-3 proteins can bind the $5^{\prime}$ untranslated region of human surfactant protein A2 mRNA, which may be liked to surfactant deficiency in neonatal respiratory distress syndrome. ${ }^{45}$ A role for YWHAZ in $\beta$ catenin signaling is potentially important in asthma pathobiology, as $\beta$-catenin is associated with regulation of TGF- $\beta$ regulated cell-cell adhesion and epithelial to mesenchyme transition, ${ }^{41}$ and $\mathrm{Wnt} / \beta$-catenin signaling is a significant determinant of airway remodeling in asthma. ${ }^{46}$ Our finding that TFCP2I1 and YWHAZ may be associated with coincident allergic pathobiology in lung tissues and BALF suggests that the initiation and progression of epithelial barrier disruption and tissue remodeling result from an integrated network of pathways that are associated, in part, with TGF- $\beta$ and Wnt $/ \beta$ catenin.

Limitations. Interpretation of our study is limited by a number of factors. Though we carefully controlled collection methods to reduce variability, such as minimizing lung tissue damage caused by collecting BALF, we cannot discount that BALF samples included a small fraction of cellular proteins. In addition, though collecting lavage fluid does remove immune cells from the airways, there is a resident population of immune cells found throughout the lung tissue that is known to match the proportions found in BALF. ${ }^{47}$ We also only collected samples at one time point ( $48 \mathrm{~h}$ after the final allergen challenge), a strategic choice, as this is when lung dysfunction is greatest. Thus, our work provides only a snapshot of the proteome response, and future studies should adopt a design to assess temporal patterns of the response to HDM challenge. We also only used a single allergen, HDM, but this was a strategic selection as HDM is a common human aeroallergen and has a complex composition that includes multiple allergens, proteases, and toxins, therefore offers the advantage of inducing inflammation of a broad profile. ${ }^{7,8}$

Summary. We characterized the proteome of lung tissues and BALF from HDM-challenged mice that mimic allergic asthma pathophysiology. Using matched samples from individual animals, our work reveals that lung tissue and BALF proteomes are diverse, and that integrating both datasets reveals additional novel biological processes and protein interaction nodes, in particular highlighting transcriptional regulation as a key integrating parameter for coincident pathobiology in lung tissues and extracellular spaces of the lung. This work provides a resource and approach for identifying new proteins and pathways and a basis to interrogate interactions between sample compartments to 
identify mechanisms for airway pathophysiology and, perhaps, new targets for developing therapeutic approaches.

\section{METHODS}

Animal Experiments. Murine HDM Allergen Challenge. All animal experiments were planned and performed following the approved protocols and guidelines of the animal ethics board at the University of Manitoba. Female, 8-week old BALB/c mice (6-8 weeks, $n=3)$ were intranasally challenged with HDM ( $25 \mu \mathrm{g}$ per mouse, $35 \mu \mathrm{L}$ saline) five times a week for 2 weeks (Supporting Information, Figure S4). HDM extract (Greer Labs; Lenoir, NC) was prepared in sterile phosphatebuffered saline ( $\mathrm{pH} 7.4$; Life Technologies; Waltham, MA). The HDM extract we used contained 36000 endotoxin units (EU; $7877 \mathrm{EU} / \mathrm{mg}$ of protein or $196.9 \mathrm{EU} /$ dose) and $4.9 \%$ Der $\mathrm{p} 1$ protein per vial.

Lung Function, Inflammatory Differential Cell Counts, and Sample Collection. Lung function was performed $48 \mathrm{~h}$ after the last HDM challenge. Mice were anesthetized with sodium pentobarbital $(90 \mathrm{mg} / \mathrm{kg})$ given intraperitoneally and tracheotomized with a 20 -gauge polyethylene catheter. The catheter was connected to a flexiVent small animal ventilator (Scireq; Montréal, Canada) and mechanically ventilated with a tidal volume of $10 \mathrm{~mL} / \mathrm{kg}$ body weight, 150 times $/ \mathrm{min}$. Forced oscillation technique and positive end expiratory pressure of 3 $\mathrm{cm} \cdot \mathrm{H}_{2} \mathrm{O}$ was used for the entire study. A nebulized methacholine $(\mathrm{MCh})$ challenge $(0-50 \mathrm{mg} / \mathrm{mL})$ was administered to assess concentration-dependent response of the respiratory mechanics. Values for each parameter (newtonian resistance, $R_{\mathrm{n}}$; peripheral tissue damping, $G$; tissue elastance, $H$; total resistance, $R$ ) were calculated as the peak of all 12 perturbation cycles performed after each MCh challenge. Statistical analysis was conducted using a two-way nested analysis of variance (ANOVA) with Tukey's multiple comparison and false discovery rate (FDR) correction (performed in $R$ ).

Post lung function measurement, lungs were lavaged with $1.0 \mathrm{~mL}$ of saline two times, for a total of $2 \mathrm{~mL}$ containing $0.1 \%$ ethylenediaminetetraacetic acid (EDTA; Sigma-Aldrich; St. Louis, MO). BALF was centrifuged to collect the immune cell pellet $\left(1000 \mathrm{~g}, 10 \mathrm{~min}, 4^{\circ} \mathrm{C}\right)$ and the supernatant was collected and aliquoted prior to flash freezing in liquid nitrogen and storage at $-80{ }^{\circ} \mathrm{C}$. The immune cell pellet was resuspended in saline and the total immune cell count was estimated using a hemocytometer. For differential counts, cells were stained with a modified Wright-Giemsa stain (HEMA 3 STAT PACK, Fisher Scientific; Waltham, MA). Cell distribution was analyzed by manually identifying and counting eosinophils, neutrophils, macrophages, and lymphocytes in six randomly chosen fields of view examined under a light microscope at 200× magnification. Post BAL, lung tissues from the left lung and half the right lung were portioned $(\sim 35 \mathrm{mg} /$ each), flashfrozen in liquid nitrogen, and stored at $-80{ }^{\circ} \mathrm{C}$.

Preparation of Lung Tissues. A randomly selected portion of lung tissue was thawed and weighed before washing the lung tissue of residual blood contamination. The lung tissue was submerged in a $15 \mathrm{~mL}$ centrifuge tube containing 15 $\mathrm{mL}$ of PBS $\left(-\mathrm{CaCl}_{2},-\mathrm{MgCl}_{2}, \mathrm{pH} 7.4\right.$; Invitrogen, Waltham, MA) with protease/phosphatase inhibitors and mixed in an end-over-end mixer $\left(4{ }^{\circ} \mathrm{C}, 30 \mathrm{~min}\right)$. Inhibitors including phenylmethylsulfonyl fluoride (PMSF, $100 \mathrm{mM}$ stock), phosphatase inhibitor cocktail 2 (Sigma-Aldrich; St. Louis, $\mathrm{MO}$ ), and protease inhibitor (Sigma-Aldrich) each at 1:100 dilution. The tissue surface was cut with scissors to increase the effectiveness of the lung tissue wash.

Tissues were homogenized using a TissueRuptor (Qiagen; Venlo, Netherlands) in siliconized centrifuge tubes (Thomas Scientific; Swedesboro, NJ) containing ice-cold lysis buffer. Lysis buffer composition: $150 \mathrm{mM} \mathrm{NaCl}$ (Fisher Scientific), 50 $\mathrm{mM}$ Tris-HCL (pH 7.5; Fisher Scientific, Waltham, MA), 5\% glycerol (Sigma-Aldrich), 1\% sodium deoxycholate (SigmaAldrich), $1 \%$ benzonase ( $25 \mathrm{U} / \mu \mathrm{L}$; Merck,; Kenilworth, NJ), $1 \%$ sodium dodecyl sulfate (SDS; Fisher Scientific), protease inhibitor cocktail 2 (1:100 dilution, Sigma-Aldrich), $1 \mathrm{mM}$ PMSF (Sigma-Aldrich), phosphatase inhibitor cocktail 2 (1:100 dilution, Sigma-Aldrich), and $2 \mathrm{mM} \mathrm{MgCl}_{2}$ (Fisher Scientific) built up with molecular grade water (Invitrogen). Cell-free supernatant $\left(21000 \mathrm{~g}, 10 \mathrm{~min}, 4{ }^{\circ} \mathrm{C}\right.$, no break) was incubated at room temperature for $30 \mathrm{~min}$ to permit benzonase activity before storage at $-80{ }^{\circ} \mathrm{C}$. All chemicals used for tissue preparation were of molecular/electrophoresis grade.

Assessment of Protein Extraction Proficiency. From each randomly chosen portion of frozen lung tissue, our protein extraction process yielded an average of $1.146 \mathrm{mg}$ of total protein ( $\mu \mathrm{BCA}$ protein assay; Pierce; Waltham, MA). Our rationale for randomizing our selection for a portion of frozen lung tissues resides in the heterogeneous nature of allergen-induced airway inflammation throughout the lung, there is no region of the lung where the extent of allergen exposure can induce uniform changes across animals. BALF yielded an average of $600 \mu \mathrm{g}$ of total protein $(100 \mu \mathrm{g}$ per 250 $\mu \mathrm{L}$ aliquot) per mouse ( $\mu \mathrm{BCA}$, Pierce). A coomassie blue stained (GelCode, Invitrogen) gradient LDS-PAGE (NuPAGE; Invitrogen) was imaged (ChemiDock, Bio-Rad) to qualitatively assess total protein molecular weight diversity of both lung tissue (Supporting Information, Figure S5A) and BALF (Supporting Information, Figure S5B) protein lysates. No high abundance protein depletion methods were employed to reduce potential elimination bias, as shown by the dark band at $\sim 66.5 \mathrm{kDa}$ approximating the molecular weight of albumin (Supporting Information, Figure S5A,B).

Replicate analysis comparing both technical and biological replicates used mouse BALF HDM samples in parallel protein filter-assisted sample preparation (FASP) procedures. BALF was divided into equal parts ( $100 \mu \mathrm{g}$ of total protein each) and processed individually and in parallel through our FASP protocol. Our results show that technical variation (Supporting Information, Figure S5E) is lower than our biological variation (Supporting Information, Figure S5F) and therefore we negated the use of technical replicates for our proteomic analysis.

Filter-Assisted Sample Preparation (FASP) of Lung Tissues and BALF. We modified a previously used FASP protocol for use in lung tissues. ${ }^{48}$ Tissue homogenate $(300 \mu \mathrm{g}$ total protein) was supplemented with dithiothreitol (DTT; final concentration $100 \mathrm{mM}$ ) before boiling for $5 \mathrm{~min}$, cooled, and supernatant $\left(21000 \mathrm{~g}, 10 \mathrm{~min}, 4{ }^{\circ} \mathrm{C}\right.$, no break) was collected. Tissue homogenates were built with urea buffer $(8$ $\mathrm{M}$ urea, $100 \mathrm{mM}$ Tris) to $800 \mu \mathrm{L}$ before loading onto $30 \mathrm{kDa}$ molecular weight cutoff filters (Amicron Ultra 0.5; Milipore; Burlington, MA). Once samples were loaded (10 000g, $10 \mathrm{~min}$, room temperature), columns were washed twice with urea buffer $(450 \mu \mathrm{L} ; 10000 \mathrm{~g}, 10 \mathrm{~min}$, room temperature $)$. Columns were alkylated $(400 \mu \mathrm{L}, 50 \mathrm{mM}$ iodoacidimide in urea buffer; Sigma-Aldrich) for $45 \mathrm{~min}$ (protected from light, room 
temperature) before being stopped by the addition of DTT $(20 \mathrm{mM})$ followed by centrifugation $(13000 \mathrm{~g}, 10 \mathrm{~min}$, room temperature). Columns were washed twice $(450 \mu \mathrm{L}$ of urea buffer) before drying the column $(14000 \mathrm{~g}, 15 \mathrm{~min}$, room temperature). Trypsin (Trypsin Gold; Promega; Madison, WI) was suspended in digestion buffer $\left(50 \mathrm{mM}\right.$ Tris, $2 \mathrm{mM} \mathrm{CaCl}_{2}$, LC-MS water) and was added to the column at a protein/ trypsin ratio of 50:1. Samples were incubated under shaking conditions at $37{ }^{\circ} \mathrm{C}$ for $16 \mathrm{~h}$ before being halted by the addition of trifluoroacetic acid (TFA; $1 \%$ final concentration) and placing the samples at $4{ }^{\circ} \mathrm{C}$. Columns were incubated (400 $\mu \mathrm{L}, 50 \%$ methanol, $5 \mathrm{~min}$ ) prior to being eluted (10 000g, 10 min, room temperature). A final wash of the column was also added to the eluted sample ( $300 \mu \mathrm{L} 15 \%$ acetonitrile). Frozen samples $\left(-80^{\circ} \mathrm{C}\right)$ were lyophilized via speed vac and stored at $-80{ }^{\circ} \mathrm{C}$.

Mouse BALF samples were processed in a similar manner to that of mouse lung tissues with some minor changes to the protocol. We resuspended $100 \mu \mathrm{g}$ of total protein from the thawed BALF supernatant in urea buffer $(450 \mu \mathrm{L}$; containing $100 \mathrm{mM}$ DTT final concentration) under mixing conditions for $1 \mathrm{~h}$ (room temperature) before loading onto columns.

Peptide Desalting by Reverse-Phase One-Dimensional Liquid Chromatography (1D-HPLC). Warmed samples were resuspended in $800 \mu \mathrm{L}$ of TFA $(0.5 \%$, LC-MS grade water) and centrifuged $\left(21000 \mathrm{~g}, 10 \mathrm{~min}, 4{ }^{\circ} \mathrm{C}\right)$ to check for undissolved peptides. BALF was loaded onto a C18 column (Luna $10 \mu \mathrm{M}$ C18(2), $100 \AA$, $50 \times 4.6 \mathrm{~mm}^{2}$; Phenomenex, Torrance, CA), while tissue lysate samples were loaded onto a separate C18 column (Phenomenex) offline. Column efficiency and elution conditions were tested using a specialized six peptide solution prior to starting the samples. ${ }^{49}$ Samples were fractionated across six $1.5 \mathrm{~mL}$ tubes and were collected at a flow rate of $500 \mu \mathrm{L} / \mathrm{min}$ with an additional $30 \mathrm{~s}$ before and after the eluted peptide spectra were detected. Manual loading was used with no gradient. Samples were frozen at $-80{ }^{\circ} \mathrm{C}$. An Agilent 1110 HPLC System using ChemStation for Control and Data Analysis (Santa Clara, CA) was used to analyze the chromatograms from reverse-phasehigh pressure liquid chromatography (HPLC).

Reconstitution of Samples and Liquid Chromatography with Tandem Mass Spectrometry (LC-MS/MS) Run. Samples were desiccated by speed vac (as previously mentioned) and reconstituted in $50 \mu \mathrm{L}$ of formic acid (0.1\%). Peptide concentration was determined by UV spectrophotometry (Nanodrop Spectrophotometer 2000, Thermofisher) at $280 \mathrm{~nm}$. Peptides $(2 \mu \mathrm{g})$ were diluted in formic acid $(0.1 \%)$ and injected into an online LC-MS/MS workflow (500 nL/ min) using a $3 \mathrm{~h}$ gradient run using a Sciex TripleTOF 5600 instrument (Sciex; Framingham, MA). Raw spectra files were converted into Mascot Generic File format (MGF) for protein identification using the tools bundled by the manufacturer. All chemicals used were mass spec grade.

Data Processing. The MGF files were processed by X! Tandem $^{50}$ against single-missed-cleavage tryptic peptides from the Mus musclus Uniprot database (16704 proteins). The following X!Tandem search parameters were used: 20 and 50 ppm mass tolerance for parent and fragment ions, respectively; constant modification of Cys with iodoacetamide; default set post-translational modifications: oxidation of Met, Trp; Nterminal cyclization at $\mathrm{Q} \ln , \mathrm{Cys} ; \mathrm{N}$-terminal acetylation, phosphorylation (Ser, Thr, Tyr), deamidation (Asn and Gln); and an expectation value cutoff of $\log _{\mathrm{e}}<-1$ for both proteins and peptides. Each MS run yielded a list of protein expression values in a $\log _{2}$ scale, quantified based on their member peptide MS2 fragment intensity sums. Quantified proteins were detected if at least two nonredundant (unique) peptides with identification scores $\log _{\mathrm{e}}<1.5$ each were identified.

Data Acquisition: Replication, Randomization, and Blocking. To homogenize the variance in our studies and avoid confounding technical variability with the biological question, we randomized the order of all our experimental sample work-up. In each treatment group, biological variability was captured with three replicate animals. Moreover, in the case of LC/MS experiments, the sample order was randomized to ameliorate effects related to instrumental drift and other variations. The hierarchical order for the magnitude of variability in many omics experiments is well-documented and it is this principle that guided our data acquisition strategy. ${ }^{51}$

Immunoblotting Validation of Proteomics. Protein targets were selected based upon proteomic enrichment after HDM exposure (hypergeometric model with FC > = 2, p.adj < $=0.05$, Benjamini-Hochberg multiple comparison) and by antibody availability. Immunoblotting was performed, as previously mentioned. ${ }^{52}$ Briefly, sodium dodecyl sulfatepolyacrylamide gel electrophoresis (SDS-PAGE) was performed on the same protein samples used for proteomic analysis. Once $20 \mu \mathrm{g}$ (total protein) of each sample was transferred onto nitrocellulose membranes, blots were stained with Ponceau $S$ total protein stain (Sigma) prior to blocking at room temperature ( $5 \%$ skim milk, Tris-buffered saline (TBST) $0.2 \%, 2 \mathrm{~h}, \mathrm{RT})$ and immunoblotted overnight at $4{ }^{\circ} \mathrm{C}(1 \%$ milk, TBST $0.2 \%)$. After washing $(3 \times 15 \mathrm{~min}$, TBST $0.2 \%)$, secondary antibody was added (Goat anti-rabbit HRP, 1\% milk, TBST $0.2 \%, 2$ h, RT, 1:5000; Sigma). Once washed, blots were flooded with ECL (Amersham; GE Healthcare, Chicago, IL) and bands were detected using a chemiluminescent film (Hyperfilm ECL; GE Healthcare). Semiquantitative densitometric analysis was performed using a scanned film and AlphaEase FC software (Alpha Innotech, San Leandro, CA). Antibodies used: FDPS (PA5-28228, 1:1000; Thermofisher), ARG1 (ab91279, 1:1000; Abcam, Cambridge, U.K.), and CLCA1 (ab180851, 1:2500; Abcam). To measure average protein content, pooled samples (Nä̈e Pool and HDM Pool) were prepared by combining equal protein content across biological replicates to equal $20 \mu \mathrm{g}$.

Bioinformatics and Statistical Analysis. Our raw data dataset $(n=2675)$ across treatment groups: allergen-naive $(n$ $=1996)$ and HDM exposed $(n=2502)$. To limit the number of comparison, we employed strict filtering criteria; we selected only proteins that are detected in both the compartments, i.e., we kept only those proteins that are identified in all replicates of at least one treatment (HDM or naive). Together this dataset is referred to the Integrated Tissue-BALF dataset (ITB, $n=1237$ ).

All of the data analyses were conducted in the $R$, version 3.6.1 (Action of the Toes), environment using in-house written code employing packages: limma (v3.42.2), vsn (v3.54.0), ggplot2 (v3.3.2), DEP (v1.8.0), and pheatmap (v1.0.12). The stepwise workflow for the analyses was as follows.

Data Preprocessing and Filtering and Normalization. The BALF proteomic measurement of one HDM-exposed mouse (labeled \#379) was found to be missing over $80 \%$ of Uniprot IDs compared to other allergen-exposed BALF samples. This 
sample was also found to be a technical outlier on examination of within group clusters visualized by principal component analysis (PCA). This sample was therefore excluded from all downstream analyses leaving a total of 13 samples to comprise our full dataset $(n=13)$ from which our results are observed (Figure S2B,C). To retain a robust biological signature for the remaining samples, we instituted a data filtering criterion in which we retained proteins whose signal was identified in at least 2 out of 3 technical replicates (or at least 3 out of 4 for HDM BALF) of at least one condition. This then allowed us to impute the rest of the missing values using the $k$-nearest neighbour (knn) imputation method. To proportionally compare the protein abundances from lung tissue and BALF samples, we performed background correction and normalization by variance stabilizing normalization (vsn). ${ }^{53}$ Using this approach, the variance of the dataset remains nearly constant over the whole dynamic range of our proteomic samples.

Exploratory Data Analysis and Differential Analysis (LIMMA). To visualize the (dis)similarities between our proteomics measurements of the samples under different conditions, we used principal component analysis (PCA) as a dimension reduction technique. The variance of all of the sample measurements for each protein was calculated, and the top 500 proteins with the greatest variance were selected. We examined the relationship among samples by projecting the original measurements on the two PCs that account for over $80 \%$ of the data variance and plotted these projections, as shown in Figures $1 \mathrm{~A}$ and $3 \mathrm{~A}$.

Moreover, to identify significantly enriched or depleted proteins we performed differential abundance analysis on the vsn-normalized data using the linear models for microarray data (LIMMA) approach. ${ }^{34}$ We used a statistical threshold of $\log _{2}$ fold changes $\geq 1$ and reported FDR-adjusted $p$-values using Benjamini-Hochberg multiple comparison corrections. The threshold for statistical significance was p.adj $\leq 0.05$. The cutoff criterion for all LIMMA results is uniform across this study.

Data Visualization. To identify the distribution of individual protein IDs across groups, proportional area Venn diagrams were constructed using the eulerr R package (v6.1.0).

Heatmaps were constructed to identify the distribution of protein IDs across conditions. These heatmaps were constructed using either normalized expression values from LIMMA (Figures 1D and 2B) or using a presence/absence classification to identify unique protein IDs (Figure 1B). In addition, grouping analysis ( $k$-means clustering) was constructed for each heatmap identifying groupings across both protein ID and sample conditions. Figures were generated using the pheatmap package in $\mathrm{R}$ (v1.0.12). Histograms, bargraphs, boxplots, line plots, and linear regressions were constructed using DataGraph (DataGraph v4.5, Visual Data Tools, Inc., Chapel Hill, NC, https://www.visualdatatools. com/).

Functional Analyses. Using the Gene Ontology database in InnateDB, we first performed over-representation analysis using the default parameters: hypergeometric model with Benjamini-Hochberg multiple comparison correction. ${ }^{55}$ To retain nonredundant biological processes, we used Revigo with a similarity search pattern of $0.5 .^{56}$ The statistical significance of biological pathway enrichment was set at p.adj $\leq 0.05$. Moreover, we performed the further functional analysis by conducting a protein-protein interaction networks analysis (NetworkAnalyst) and Biological Pathway Assessment (Re- actome). Protein-protein interaction network analysis was performed by inputting Uniprot IDs into NetworkAnalyst (v3.0, accessed April 2020) and analyzed using the InnateDB protein interaction network database. ${ }^{55,57}$ The top first-order network (by the number of connections) was selected for further analysis. Within each network, the most interconnected protein nodes (from the input Uniprot ID list) that are unique to either BALF or tissue were selected and subset from the larger networks to examine direct connections between the selected nodes. Biological pathway analysis was then performed using the Reactome database module inside NetworkAnalyst (hypergeometric model with p.adj $\leq 0.05$ by Benjamini-Hochberg multiple comparison). The protein UBC was not detected in any of our analyses and was removed from all protein-protein interaction analyses.

Dataset Preparation for Predicting Lung Tissues/BALF Interactions. We first integrated the 362 proteins that were uniquely enriched by HDM challenge in lung tissues with the 311 proteins that were uniquely enriched after allergen challenge in BALF. After excluding the 49 proteins that are shared between the two datasets, we input the lung tissue $(n=$ $311)$, BALF $(n=262)$, and combined $(n=573)$ datasets into NetworkAnalyst. To focus on the nodes that are the most interconnected between the BALF and lung tissue datasets, we filtered the combined dataset to include proteins with five or more first-order interactions. ${ }^{58}$ From the 37 proteins that met this criterion ( 12 from lung tissue, 25 from BALF), we selected the top 5 proteins from the BALF and lung tissue dataset that become enriched (\% enrichment) in the combined dataset. Selecting the top 5 proteins from each dataset was essential to focus network complexity on the top contributors to protein interactions.

Data Storage. We have made all proteomic data freely available in the Mass Spectrometry Interactive Virtual Environment (MassIVE) database housed at UCSD under the id MSV000086003.

\section{ASSOCIATED CONTENT}

\section{st Supporting Information}

The Supporting Information is available free of charge at https://pubs.acs.org/doi/10.1021/acsomega.0c04269.

HDM exposure induces altered lung function and increased inflammatory cell counts (Figure S1A, S1B); summary of MS/MS analysis from X!Tandem informatic pipeline (Table S2A, Figure S2B,C); experimental workflow (Figure S4); proteomic quality control and reproducibility tests indicate proteomic variability resides in biological and not technical replicates (Figure S5A-F) (PDF)

Listings of protein ID's across Venn diagrams (File 1) (XLSX)

\section{AUTHOR INFORMATION}

\section{Corresponding Author}

Andrew J. Halayko - Department of Physiology \& Pathophysiology, University of Manitoba, Winnipeg, Manitoba R3T 2N2, Canada; DEVOTION Network, Winnipeg, Manitoba R3E 3P4, Canada; Biology of Breathing Group, Children's Hospital Research Institute of Manitoba, Winnipeg, Manitoba R3E 3P4, Canada; Canadian Respiratory Research Network, Ottawa, Ontario K2E 7V7, 
Canada; ๑ orcid.org/0000-0002-7865-4552;

Email: andrew.halayko@umanitoba.ca

\section{Authors}

Thomas H. Mahood - Department of Physiology \& Pathophysiology, University of Manitoba, Winnipeg, Manitoba R3T 2N2, Canada; DEVOTION Network, Winnipeg, Manitoba R3E 3P4, Canada; Biology of Breathing Group, Children's Hospital Research Institute of Manitoba, Winnipeg, Manitoba R3E 3P4, Canada; Canadian Respiratory Research Network, Ottawa, Ontario K2E 7V7, Canada

Christopher D. Pascoe - Department of Physiology \& Pathophysiology, University of Manitoba, Winnipeg, Manitoba R3T 2N2, Canada; DEVOTION Network, Winnipeg, Manitoba R3E 3P4, Canada; Biology of Breathing Group, Children's Hospital Research Institute of Manitoba, Winnipeg, Manitoba R3E 3P4, Canada; Canadian Respiratory Research Network, Ottawa, Ontario K2E 7V7, Canada

Tobias K. Karakach - Bioinformatics Core Laboratory, Children's Hospital Research Institute of Manitoba, Winnipeg, Manitoba R3E 3P4, Canada

Aruni Jha - Department of Physiology \& Pathophysiology, University of Manitoba, Winnipeg, Manitoba R3T 2N2, Canada; DEVOTION Network, Winnipeg, Manitoba R3E 3P4, Canada; Biology of Breathing Group, Children's Hospital Research Institute of Manitoba, Winnipeg, Manitoba R3E 3P4, Canada; Canadian Respiratory Research Network, Ottawa, Ontario K2E 7V7, Canada

Sujata Basu - Department of Physiology \& Pathophysiology, University of Manitoba, Winnipeg, Manitoba R3T 2N2, Canada; DEVOTION Network, Winnipeg, Manitoba R3E 3P4, Canada; Biology of Breathing Group, Children's Hospital Research Institute of Manitoba, Winnipeg, Manitoba R3E 3P4, Canada; Canadian Respiratory Research Network, Ottawa, Ontario K2E 7V7, Canada

Peyman Ezzati - Manitoba Centre for Proteomics and Systems Biology, Department of Internal Medicine, University of Manitoba, Winnipeg, Manitoba R3E 3P4, Canada

Victor Spicer - Manitoba Centre for Proteomics and Systems Biology, Department of Internal Medicine, University of Manitoba, Winnipeg, Manitoba R3E 3P4, Canada

Neeloffer Mookherjee - DEVOTION Network, Winnipeg, Manitoba R3E 3P4, Canada; Biology of Breathing Group, Children's Hospital Research Institute of Manitoba, Winnipeg, Manitoba R3E 3P4, Canada; Manitoba Centre for Proteomics and Systems Biology, Department of Internal Medicine and Department of Immunology, University of Manitoba, Winnipeg, Manitoba R3E 3P4, Canada; Canadian Respiratory Research Network, Ottawa, Ontario K2E 7V7, Canada

Complete contact information is available at:

https://pubs.acs.org/10.1021/acsomega.0c04269

\section{Author Contributions}

${ }^{\circ}$ T.H.M. and C.D.P. contributed equally to this work. T.H.M. performed all proteomics experiments, primary and secondary data analysis, prepared all figures and tables, and wrote the manuscript draft. C.D.P. provided supervision, guidance and assisted in the completed biostatistics and bioinformatics, design of figures, and editing and writing of the manuscript. T.K.K. provided secondary data quality control, statistical and bioinformatics support, as well as support for the design and editing of both figures and the final manuscript. A.J. and S.B. were involved with the experimental design and completion of animal studies. P.E. guided the sample preparation and performed mass spectrometry. V.S. contributed to the experimental design, primary proteomic data analysis, proteomic data quality control, and primary statistics. N.M. contributed to the experimental design and direction and edited the manuscript. A.J.H. conceived and led the design of the study, including scope of biostatistics and bioinformatics, and contributed to the writing and editing of the final manuscript.

Notes

The authors declare no competing financial interest.

\section{ACKNOWLEDGMENTS}

Work for this study was supported in part by the CIHRCanadian Respiratory Research Network (CRRN), Research Manitoba, the DEVOTION Network, and the Biology of Breathing Group, Children's Hospital Research Institute of Manitoba. T.H.M. was supported by studentships from Research Manitoba, CRRN, AllerGen NCE Inc., and Asthma Canada. C.D.P. was supported by a CIHR Banting Fellowship and a Research Manitoba Fellowship. A.J. was supported by studentships from Research Manitoba and CRRN. A.J.H. is supported through the Canada Research Chairs Program.

\section{REFERENCES}

(1) Calderón, M. A.; Linneberg, A.; Kleine-Tebbe, J.; De Blay, F.; Hernandez Fernandez de Rojas, D.; Virchow, J. C.; Demoly, P. Respiratory allergy caused by house dust mites: What do we really know. J. Allergy Clin. Immunol. 2015, 136, 38-48.

(2) Choopong, J.; Reamtong, O.; Sookrung, N.; Seesuay, W.; Indrawattana, N.; Sakolvaree, Y.; Chaicumpa, W.; Tungtrongchitr, A. Proteome, Allergenome, and Novel Allergens of House Dust Mite, Dermatophagoides farinae. J. Proteome Res. 2016, 15, 422-430.

(3) Jha, A.; Ryu, M. H.; Oo, O.; Bews, H. J.; Carlson, J. C.; Schwartz, J.; Basu, S.; Wong, C. S.; Halayko, A. J. Prophylactic benefits of systemically delivered simvastatin treatment in a house dust mite challenged murine model of allergic asthma. Br. J. Pharmacol. 2018, $175,1004-1016$.

(4) Wu, J.; Kobayashi, M.; Sousa, E. A.; Liu, W.; Cai, J.; Goldman, S. J.; Dorner, A. J.; Projan, S. J.; Kavuru, M. S.; Qiu, Y.; Thomassen, M. J. Differential proteomic analysis of bronchoalveolar lavage fluid in asthmatics following segmental antigen challenge. Mol. Cell. Proteomics 2005, 4, 1251-1264.

(5) O’Neil, S. E.; Sitkauskiene, B.; Babusyte, A.; Krisiukeniene, A.; Stravinskaite-Bieksiene, K.; Sakalauskas, R.; Sihlbom, C.; Ekerljung, L.; Carlsohn, E.; Lötvall, J. Network analysis of quantitative proteomics on asthmatic bronchi: effects of inhaled glucocorticoid treatment. Respir. Res. 2011, 12, No. 124.

(6) Burg, D.; Schofield, J. P. R.; Brandsma, J.; Staykova, D.; Folisi, C.; Bansal, A.; Nicholas, B.; Xian, Y.; Rowe, A.; Corfield, J.; Wilson, S.; Ward, J.; Lutter, R.; Fleming, L.; Shaw, D. E.; Bakke, P. S.; Caruso, M.; Dahlen, S. E.; Fowler, S. J.; Hashimoto, S.; Horváth, I.; Howarth, P.; Krug, N.; Montuschi, P.; Sanak, M.; Sandström, T.; Singer, F.; Sun, K.; Pandis, I.; Auffray, C.; Sousa, A. R.; Adcock, I. M.; Chung, K. F.; Sterk, P. J.; Djukanović, R.; Skipp, P. J.; The, U.-B. S. G. LargeScale Label-Free Quantitative Mapping of the Sputum Proteome. J. Proteome Res. 2018, 17, 2072-2091.

(7) Pascoe, C. D.; Jha, A.; Basu, S.; Mahood, T.; Lee, A.; Hinshaw, S.; Falsafi, R.; Hancock, R. E. W.; Mookherjee, N.; Halayko, A. J. The importance of reporting house dust mite endotoxin abundance: impact on the lung transcriptome. Am. J. Physiol.: Lung Cell. Mol. Physiol. 2020, 318, L1229-L1236. 
(8) Piyadasa, H.; Altieri, A.; Basu, S.; Schwartz, J.; Halayko, A. J.; Mookherjee, N. Biosignature for airway inflammation in a house dust mite-challenged murine model of allergic asthma. Biol. Open 2016, 5, $112-121$.

(9) Wypych, T. P.; Marzi, R.; Wu, G. F.; Lanzavecchia, A.; Sallusto, F. Role of $\mathrm{B}$ cells in $\mathrm{T}_{\mathrm{H}}$ cell responses in a mouse model of asthma. J. Allergy Clin. Immunol. 2018, 141, 1395-1410.

(10) Turner, D. L.; Goldklang, M.; Cvetkovski, F.; Paik, D.; Trischler, J.; Barahona, J.; Cao, M.; Dave, R.; Tanna, N.; D’Armiento, J. M.; Farber, D. L. Biased Generation and In Situ Activation of Lung Tissue-Resident Memory CD4 T Cells in the Pathogenesis of Allergic Asthma. J. Immunol. 2018, 200, 1561-1569.

(11) Svenningsen, S.; Kirby, M.; Starr, D.; Coxson, H. O.; Paterson, N. A.; McCormack, D. G.; Parraga, G. What are ventilation defects in asthma. Thorax 2014, 69, 63-71.

(12) Su, X. M.; Ren, Y.; Li, M. L.; Zhao, X.; Kong, L. F.; Kang, J. Performance evaluation of histone deacetylases in lungs of mice exposed to ovalbumin aerosols. J. Physiol. Pharmacol. 2018, 69, 265273.

(13) Ito, K.; Caramori, G.; Lim, S.; Oates, T.; Chung, K. F.; Barnes, P. J.; Adcock, I. M. Expression and activity of histone deacetylases in human asthmatic airways. Am. J. Respir. Crit. Care Med. 2002, 166, 392-396.

(14) Ito, K.; Ito, M.; Elliott, W. M.; Cosio, B.; Caramori, G.; Kon, O. M.; Barczyk, A.; Hayashi, S.; Adcock, I. M.; Hogg, J. C.; Barnes, P. J. Decreased histone deacetylase activity in chronic obstructive pulmonary disease. N. Engl. J. Med. 2005, 352, 1967-1976.

(15) Poynter, M. E.; Irvin, C. G.; Janssen-Heininger, Y. M. Rapid activation of nuclear factor-kappaB in airway epithelium in a murine model of allergic airway inflammation. Am. J. Pathol. 2002, 160, $1325-1334$

(16) Kallsen, K.; Andresen, E.; Heine, H. Histone deacetylase (HDAC) 1 controls the expression of beta defensin 1 in human lung epithelial cells. PLoS One 2012, 7, No. e50000.

(17) Redhu, N. S.; Saleh, A.; Lee, H. C.; Halayko, A. J.; Ziegler, S. F.; Gounni, A. S. IgE induces transcriptional regulation of thymic stromal lymphopoietin in human airway smooth muscle cells. J. Allergy Clin. Immunol. 2011, 128, 892.e2-896.e2.

(18) Huang, Y.; Myers, M. P.; Xu, R. M. Crystal structure of the HP1-EMSY complex reveals an unusual mode of HP1 binding. Structure 2006, 14, 703-712.

(19) Lu, J.; Gilbert, D. M. Proliferation-dependent and cell cycle regulated transcription of mouse pericentric heterochromatin. J. Cell Biol. 2007, 179, 411-421.

(20) Ezell, S. A.; Polytarchou, C.; Hatziapostolou, M.; Guo, A.; Sanidas, I.; Bihani, T.; Comb, M. J.; Sourvinos, G.; Tsichlis, P. N. The protein kinase Akt1 regulates the interferon response through phosphorylation of the transcriptional repressor EMSY. Proc. Natl. Acad. Sci. U.S.A. 2012, 109, E613-E621.

(21) Chen, J.; Chen, Q.; Wu, C.; Jin, Y. Genetic variants of the C11orf30-LRRC32 region are associated with childhood asthma in the Chinese population. Allergol. Immunopathol. 2019, 48, 390-394.

(22) Wang, T.; Cleary, R. A.; Wang, R.; Tang, D. D. Role of the adapter protein Abil in actin-associated signaling and smooth muscle contraction. J. Biol. Chem. 2013, 288, 20713-20722.

(23) Cleary, R. A.; Wang, R.; Wang, T.; Tang, D. D. Role of Abl in airway hyperresponsiveness and airway remodeling. Respir. Res. 2013, 14, No. 105.

(24) Nestor, M. W.; Cai, X.; Stone, M. R.; Bloch, R. J.; Thompson, S. M. The actin binding domain of $\beta \mathrm{I}$-spectrin regulates the morphological and functional dynamics of dendritic spines. PLoS One 2011, 6, No. e16197.

(25) Ray, S.; Chakrabarti, A. Membrane interaction of erythroid spectrin: surface-density-dependent high-affinity binding to phosphatidylethanolamine. Mol. Membr. Biol. 2004, 21, 93-100.

(26) Diakowski, W.; Sikorski, A. F. Interaction of brain spectrin (fodrin) with phospholipids. Biochemistry 1995, 34, 13252-13258.
(27) Tang, Y.; Katuri, V.; Dillner, A.; Mishra, B.; Deng, C. X.; Mishra, L. Disruption of transforming growth factor-beta signaling in ELF beta-spectrin-deficient mice. Science. 2003, 299, 574-577.

(28) Ojiaku, C. A.; Cao, G.; Zhu, W.; Yoo, E. J.; Shumyatcher, M.; Himes, B. E.; An, S. S.; Panettieri, R. A. TGF- $\beta 1$ Evokes Human Airway Smooth Muscle Cell Shortening and Hyperresponsiveness via Smad3. Am. J. Respir. Cell Mol. Biol. 2018, 58, 575-584.

(29) Keller, I. E.; Vosyka, O.; Takenaka, S.; Kloß, A.; Dahlmann, B.; Willems, L. I.; Verdoes, M.; Overkleeft, H. S.; Marcos, E.; Adnot, S.; Hauck, S. M.; Ruppert, C.; Günther, A.; Herold, S.; Ohno, S.; Adler, H.; Eickelberg, O.; Meiners, S. Regulation of immunoproteasome function in the lung. Sci. Rep. 2015, 5, No. 10230.

(30) Sixt, S. U.; Adamzik, M.; Spyrka, D.; Saul, B.; Hakenbeck, J.; Wohlschlaeger, J.; Costabel, U.; Kloss, A.; Giesebrecht, J.; Dahlmann, B.; Peters, J. Alveolar extracellular $20 \mathrm{~S}$ proteasome in patients with acute respiratory distress syndrome. Am. J. Respir. Crit. Care Med. 2009, 179, 1098-1106.

(31) Elliott, P. J.; Pien, C. S.; McCormack, T. A.; Chapman, I. D.; Adams, J. Proteasome inhibition: A novel mechanism to combat asthma. J. Allergy Clin. Immunol. 1999, 104, 294-300.

(32) Sixt, S. U.; Peters, J. Extracellular alveolar proteasome: possible role in lung injury and repair. Proc. Am. Thorac. Soc. 2010, 7, 91-96.

(33) Yamazaki, T.; Masuda, J.; Omori, T.; Usui, R.; Akiyama, H.; Maru, Y. EphA1 interacts with integrin-linked kinase and regulates cell morphology and motility. J. Cell Sci. 2009, 122, 243-255.

(34) Jeon, S. G.; Lee, C. G.; Oh, M. H.; Chun, E. Y.; Gho, Y. S.; Cho, S. H.; Kim, J. H.; Min, K. U.; Kim, Y. Y.; Kim, Y. K.; Elias, J. A. Recombinant basic fibroblast growth factor inhibits the airway hyperresponsiveness, mucus production, and lung inflammation induced by an allergen challenge. J. Allergy Clin. Immunol. 2007, $119,831-837$.

(35) Liu, F.; Hata, A.; Baker, J. C.; Doody, J.; Cárcamo, J.; Harland, R. M.; Massagué, J. A human Mad protein acting as a BMP-regulated transcriptional activator. Nature 1996, 381, 620-623.

(36) Nyman, T.; Trésaugues, L.; Welin, M.; Lehtiö, L.; Flodin, S.; Persson, C.; Johansson, I.; Hammarström, M.; Nordlund, P. The crystal structure of the Dachshund domain of human SnoN reveals flexibility in the putative protein interaction surface. PLoS One 2010, 5, No. e12907.

(37) He, J.; Tegen, S. B.; Krawitz, A. R.; Martin, G. S.; Luo, K. The transforming activity of Ski and SnoN is dependent on their ability to repress the activity of Smad proteins. J. Biol. Chem. 2003, 278, 30540-30547.

(38) Meng, X.; Lu, P.; Bai, H.; Xiao, P.; Fan, Q. Transcriptional regulatory networks in human lung adenocarcinoma. Mol. Med. Rep. 2012, 6, 961-966.

(39) Fattouh, R.; Midence, N. G.; Arias, K.; Johnson, J. R.; Walker, T. D.; Goncharova, S.; Souza, K. P.; Gregory, R. C.; Lonning, S.; Gauldie, J.; Jordana, M. Transforming growth factor-beta regulates house dust mite-induced allergic airway inflammation but not airway remodeling. Am. J. Respir. Crit. Care Med. 2008, 177, 593-603.

(40) Yamaguchi, Y.; Yonemura, S.; Takada, S. Grainyhead-related transcription factor is required for duct maturation in the salivary gland and the kidney of the mouse. Development 2006, 133, 47374748 .

(41) Giangreco, A.; Lu, L.; Vickers, C.; Teixeira, V. H.; Groot, K. R.; Butler, C. R.; Ilieva, E. V.; George, P. J.; Nicholson, A. G.; Sage, E. K.; Watt, F. M.; Janes, S. M. $\beta$-Catenin determines upper airway progenitor cell fate and preinvasive squamous lung cancer progression by modulating epithelial-mesenchymal transition. J. Pathol. 2012, 226, 575-587.

(42) Chen, C. H.; Chuang, S. M.; Yang, M. F.; Liao, J. W.; Yu, S. L.; Chen, J. J. A novel function of YWHAZ/ $\beta$-catenin axis in promoting epithelial-mesenchymal transition and lung cancer metastasis. Mol. Cancer Res. 2012, 10, 1319-1331.

(43) Weerasekara, V. K.; Panek, D. J.; Broadbent, D. G.; Mortenson, J. B.; Mathis, A. D.; Logan, G. N.; Prince, J. T.; Thomson, D. M.; Thompson, J. W.; Andersen, J. L. Metabolic-stress-induced rearrangement of the $14-3-3 \zeta$ interactome promotes autophagy via a ULK1- 
and AMPK-regulated 14-3-3 $\zeta$ interaction with phosphorylated Atg9. Mol. Cell. Biol. 2014, 34, 4379-4388.

(44) Lee, J. J.; Lee, J. S.; Cui, M. N.; Yun, H. H.; Kim, H. Y.; Lee, S. H.; Lee, J. H. BIS targeting induces cellular senescence through the regulation of 14-3-3 zeta/STAT3/SKP2/p27 in glioblastoma cells. Cell Death Dis. 2014, 5, No. e1537.

(45) Noutsios, G. T.; Ghattas, P.; Bennett, S.; Floros, J. 14-3-3 isoforms bind directly exon B of the 5 -UTR of human surfactant protein A2 mRNA. Am. J. Physiol.: Lung Cell. Mol. Physiol. 2015, 309, L147-L157.

(46) Kumawat, K.; Koopmans, T.; Gosens, R. $\beta$-catenin as a regulator and therapeutic target for asthmatic airway remodeling. Expert Opin. Ther. Targets 2014, 18, 1023-1034.

(47) Movassagh, H.; Shan, L.; Mohammed, A.; Halayko, A. J.; Gounni, A. S. Semaphorin 3E Deficiency Exacerbates Airway Inflammation, Hyperresponsiveness, and Remodeling in a Mouse Model of Allergic Asthma. J. Immunol. 2017, 198, 1805-1814.

(48) Wiśniewski, J. R.; Zougman, A.; Nagaraj, N.; Mann, M. Universal sample preparation method for proteome analysis. Nat. Methods 2009, 6, 359-362.

(49) Krokhin, O. V.; Spicer, V. Peptide retention standards and hydrophobicity indexes in reversed-phase high-performance liquid chromatography of peptides. Anal. Chem. 2009, 81, 9522-9530.

(50) Craig, R.; Beavis, R. C. TANDEM: matching proteins with tandem mass spectra. Bioinformatics 2004, 20, 1466-1467.

(51) Karakach, T. K.; Wentzell, P. D.; Walter, J. A. Characterization of the measurement error structure in $1 \mathrm{D} 1 \mathrm{H}$ NMR data for metabolomics studies. Anal. Chim. Acta 2009, 636, 163-174.

(52) Tran, T.; McNeill, K. D.; Gerthoffer, W. T.; Unruh, H.; Halayko, A. J. Endogenous laminin is required for human airway smooth muscle cell maturation. Respir. Res. 2006, 7, No. 117.

(53) Välikangas, T.; Suomi, T.; Elo, L. L. A systematic evaluation of normalization methods in quantitative label-free proteomics. Brief Bioinform. 2018, 19, 1-11.

(54) Ritchie, M. E.; Phipson, B.; Wu, D.; Hu, Y.; Law, C. W.; Shi, W.; Smyth, G. K. limma powers differential expression analyses for RNA-sequencing and microarray studies. Nucleic Acids Res. 2015, 43, No. e47.

(55) Breuer, K.; Foroushani, A. K.; Laird, M. R.; Chen, C.; Sribnaia, A.; Lo, R.; Winsor, G. L.; Hancock, R. E.; Brinkman, F. S.; Lynn, D. J. InnateDB: systems biology of innate immunity and beyond-recent updates and continuing curation. Nucleic Acids Res. 2013, 41, D1228D1233.

(56) Supek, F.; Bošnjak, M.; Škunca, N.; Šmuc, T. REVIGO summarizes and visualizes long lists of gene ontology terms. PLoS One 2011, 6, No. e21800.

(57) Zhou, G.; Soufan, O.; Ewald, J.; Hancock, R. E. W.; Basu, N.; Xia, J. NetworkAnalyst 3.0: a visual analytics platform for comprehensive gene expression profiling and meta-analysis. Nucleic Acids Res. 2019, 47, W234-W241.

(58) Vallabhajosyula, R. R.; Chakravarti, D.; Lutfeali, S.; Ray, A.; Raval, A. Identifying hubs in protein interaction networks. PLoS One 2009, 4, No. e5344. 